Pacific

Journal of

Mathematics

\title{
METAPLECTIC TORI OVER LOCAL FIELDS
}

MARTIN H. WEISSMAN 


\title{
METAPLECTIC TORI OVER LOCAL FIELDS
}

\author{
MARTIN H. WEISSMAN
}

\begin{abstract}
Smooth irreducible representations of tori over local fields have been parameterized by Langlands, using class field theory and Galois cohomology. This paper extends this parameterization to some central extensions of such tori, which arise naturally in the setting of nonlinear covers of reductive groups.
\end{abstract}

1. Introduction 169

2. Background 171

3. Genuine representations of metaplectic tori 175

4. Split tori 177

5. Unramified tori 180

6. Pseudospherical and pseudotrivial representations $\quad 192$

7. Tori over $\mathbb{R} \quad 196$

Acknowledgments 199

References 199

\section{Introduction}

Motivation. Let $\boldsymbol{T}$ be an algebraic torus over a local field $F$; let $T=\boldsymbol{T}(F)$. Let $L / F$ be a finite Galois extension over which $T$ splits, with $\Gamma=\operatorname{Gal}(L / F)$. Let $\mathscr{L}(T)$ denote the group of continuous characters of $T$ with values in $\mathbb{C}^{\times}$. In a preprint from 1968, now appearing as [Langlands 1997] (see [Labesse 1985]), Langlands proves the following:

Theorem 1.1. There is a natural isomorphism

$$
\mathscr{X}(T) \cong H_{c}^{1}\left(W_{L / F}, \hat{\mathscr{T}}\right),
$$

where $\mathcal{W}_{L / F}$ denotes the Weil group of $L / F, \hat{\mathfrak{T}}$ denotes the complex dual torus of $\boldsymbol{T}$, and $H_{c}^{1}$ denotes the continuous group cohomology.

Keywords: metaplectic, torus, Langlands, local field. 
We may consider $\boldsymbol{T}$ as a sheaf of groups, on the big Zariski site over $F$. In addition, we may consider $\boldsymbol{K}_{2}$ as such a sheaf, using Quillen's algebraic K-theory. Let $\boldsymbol{T}^{\prime}$ be a central extension of $\boldsymbol{T}$ by $\boldsymbol{K}_{2}$ in the category of sheaves of groups on the big Zariski site over $F$. Such objects are introduced and studied extensively by Brylinski and Deligne [2001].

Let $T^{\prime}=\boldsymbol{T}^{\prime}(F)$ be the resulting extension of $T$ by $K_{2}=\boldsymbol{K}_{2}(F)$. If $F \neq \mathbb{C}$ and $F$ has sufficiently many $n$-th roots of unity, one may push forward the central extension $T^{\prime}$ via the Hilbert symbol to obtain a central extension $\tilde{T}$ as

$$
1 \rightarrow \mu_{n} \rightarrow \tilde{T} \rightarrow T \rightarrow 1
$$

We are interested in the set $\Phi_{\epsilon}(\tilde{T})$ of irreducible genuine representations of $\tilde{T}$, as defined in Section 3. Such representations arise frequently in the literature on "metaplectic groups", especially when considering principal series representations of nonlinear covers of reductive groups (see among others [Savin 2004; Kazhdan and Patterson 1984; Adams et al. 2007]). This paper's goal is to parameterize the set $\Im_{\epsilon}(\tilde{T})$ in a way that naturally generalizes Theorem 1.1 .

Main results. Associated to the central extension $\boldsymbol{T}^{\prime}$, Deligne and Brylinski associate two functorial invariants: an integer-valued quadratic form $Q$ on the cocharacter lattice $Y$ of $\boldsymbol{T}$, and a $\Gamma$-equivariant central extension $\tilde{Y}$ of $Y$ by $L^{\times}$. Associated to $Q$ is a symmetric bilinear form $B_{Q}: Y \otimes_{\mathbb{Z}} Y \rightarrow \mathbb{Z}$.

Define

$$
Y^{\#}=\left\{y \in Y \text { such that } B_{Q}\left(y, y^{\prime}\right) \in n \mathbb{Z} \text { for all } y^{\prime} \in Y\right\} .
$$

Similarly, define

$$
Y^{\Gamma \#}=\left\{y \in Y \text { such that } B_{Q}\left(y, y^{\prime}\right) \in n \mathbb{Z} \text { for all } y^{\prime} \in Y^{\Gamma}\right\} .
$$

Associated to the inclusion $\imath: Y^{\#} \hookrightarrow Y$ is an isogeny $\hat{\imath}: \hat{\mathscr{T}} \rightarrow \hat{\mathscr{T}}^{\#}$ of complex tori. This isogeny is also a morphism of $\mathcal{W}_{L / F}$-modules. Associated to the sequence of inclusions $Y^{\#} \subset Y^{\Gamma \#} \subset Y$ are $F$-isogenies $\boldsymbol{T}^{\#} \rightarrow \boldsymbol{T}^{\Gamma \#} \rightarrow \boldsymbol{T}$ of $F$-tori. The main results of this paper are Theorems 4.8, 5.17, and 7.7. Putting these theorems together yields the following:

Theorem 1.2. Suppose that one of these conditions is satisfied:

(1) $\boldsymbol{T}$ is a split torus.

(2) $F$ is nonarchimedean with residue field $\mathbf{f}$, the torus $\boldsymbol{T}$ splits over an unramified extension of $F$, and $n$ is relatively prime to the characteristic of $\mathbf{f}$.

(3) $F \cong \mathbb{R}$. 
Then, there exists a finite-to-one map

$$
\Phi: \Phi_{\epsilon}(\tilde{T}) \rightarrow H_{c}^{1}\left(W_{L / F}, \hat{\mathscr{T}}\right) / H_{c}^{1}\left(W_{L / F}, \hat{\mathscr{T}} \rightarrow \hat{\mathscr{T}}^{\#}\right)
$$

that intertwines canonical actions of $H_{c}^{1}\left(W_{L / F}, \hat{\mathcal{T}}\right)$. The finite fibres of this map are torsors for a finite group $\mathscr{X}\left(P^{\dagger}\right)=\operatorname{Hom}\left(P^{\dagger}, \mathbb{C}^{\times}\right)$. In the three cases above, the "packet group" $P^{\dagger}$ can be respectively described by the three conditions that

(1) $P^{\dagger}$ is trivial;

(2) $P^{\dagger}=\operatorname{Im}\left(\overline{\boldsymbol{T}}^{\Gamma \#}(\mathbf{f}) \rightarrow \overline{\boldsymbol{T}}(\mathbf{f})\right) / \operatorname{Im}\left(\overline{\boldsymbol{T}}^{\#}(\mathbf{f}) \rightarrow \overline{\boldsymbol{T}}(\mathbf{f})\right)$;

(3) $P^{\dagger}=\operatorname{Im}\left(\pi_{0} \boldsymbol{T}^{\Gamma \#}(\mathbb{R}) \rightarrow \pi_{0} \boldsymbol{T}(\mathbb{R})\right) / \operatorname{Im}\left(\pi_{0} \boldsymbol{T}^{\#}(\mathbb{R}) \rightarrow \pi_{0} \boldsymbol{T}(\mathbb{R})\right)$.

In this theorem, $H_{c}^{1}$ denotes the continuous group cohomology or hypercohomology, as discussed by Kottwitz and Shelstad [1999]. The parameterization $\Phi$ of irreducible genuine representations is not unique; rather, it depends upon the choice of a base point. The choice of this base point is a significant problem. We identify a natural class of "pseudospherical" representations, following previous authors such as [Savin 2004] and [Adams et al. 2007]. We also parameterize pseudospherical irreducible representations as a torsor for a complex algebraic torus in Section 6; perhaps more naturally, the category of pseudospherical representations can be identified with the category of modules over a "quantum dual torus".

\section{Background}

Fields and sheaves. $F$ will always denote a local field. $F_{Z a r}$ will denote the big Zariski site over $F$. By this, we mean that $F_{\text {Zar }}$ is the full subcategory of the category of schemes over $F$, whose objects are schemes of finite type over $F$, endowed with the Zariski topology. $\mathfrak{S e t}_{F}$ will denote the topos of sheaves of sets over $F_{Z a r}$, and $\mathfrak{G p}_{F}$ will denote the topos of sheaves of groups over $F_{\mathrm{Zar}}$.

Any scheme or algebraic group over $F$ will be identified with its functor of points, that is, the associated object of $\mathfrak{S e t}_{F}$ or $\mathfrak{G p}_{F}$, respectively. Quillen's K-theory [1973] yields sheaves $\boldsymbol{K}_{n}$ of abelian groups on $F_{Z \text { ar }}$. We only work with $\boldsymbol{K}_{1}$ and $\boldsymbol{K}_{2}$, viewed as objects of $\mathfrak{G}_{F}$.

For any field $L$, the group $\boldsymbol{K}_{2}(L)$ is identified as a quotient

$$
\boldsymbol{K}_{2}(L)=\frac{L^{\times} \otimes_{\mathbb{Z}} L^{\times}}{\langle x \otimes(1-x)\rangle_{1 \neq x \in L^{\times}}} .
$$

If $l_{1}, l_{2} \in L$, and $l_{1}, l_{2} \notin\{0,1\}$, then we write $\left\{l_{1}, l_{2}\right\}$ for the image of $l_{1} \otimes l_{2}$ in $\boldsymbol{K}_{2}(L)$. The bilinear form $\{\cdot, \cdot\}$ is called the universal symbol. The relation $\{x, 1-x\}=1$ implies that $\{x,-x\}=1$ for all $x \in L^{\times}$. This implies that the universal symbol is skew-symmetric. It is usually not alternating, but $\{x, x\}=\{x,-1\}$ for all $x \in L^{\times}$. Proofs of these facts can be found in [Milnor 1971, Chapter 11]. 
Local nonarchimedean fields. Suppose that $F$ is a nonarchimedean local field. Then $O_{F}$ will denote the valuation ring of $F$, and $\mathbf{f}$ the residue field of $O_{F}$. We let $p$ denote the characteristic of $\mathbf{f}$ and assume that the value group of $F$ is $\mathbb{Z}$. We let $q$ denote the cardinality of $\mathbf{f}$.

There is a canonical short exact sequence

$$
1 \rightarrow \mathrm{O}_{F}^{\times} \rightarrow F^{\times} \rightarrow \mathbb{Z} \rightarrow 1
$$

of abelian groups, given by inclusion and valuation. It is sometimes convenient to split this sequence of abelian groups by choosing a uniformizing element $\varpi \in F^{\times}$. However, our main results do not depend on which uniformizing element is chosen.

Reduction yields another canonical short exact sequence

$$
1 \rightarrow \mathrm{O}_{F}^{\times 1} \rightarrow \mathrm{O}_{F}^{\times} \rightarrow \mathbf{f}^{\times} \rightarrow 1
$$

This sequence is split by the Teichmüller lifting $\Theta: \mathbf{f}^{\times} \rightarrow \mathbb{O}_{F}^{\times}$.

The Weil group. We let $\mathcal{W}_{F}$ denote a Weil group of $F$ as in [Tate 1979]. In particular, we follow Tate's choices and normalize the reciprocity isomorphism rec: $F^{\times} \rightarrow \mathcal{W}_{F}^{a b}$ of nonarchimedean local class field theory so that uniformizing elements of $F^{\times}$act as the geometric Frobenius via rec.

When $L$ is a finite Galois extension of $F$, we continue to follow [Tate 1979] and define $\mathcal{W}_{L / F}=W_{F} / \overline{\left[\mathcal{W}_{L}, \mathscr{W}_{L}\right]}$. There is then a short exact sequence

$$
1 \rightarrow L^{\times} \rightarrow \mathcal{W}_{L / F} \rightarrow \operatorname{Gal}(L / F) \rightarrow 1 .
$$

The Hilbert symbol. We say that $F$ has enough $n$-th roots of unity if $\boldsymbol{\mu}_{n}(F)$ has $n$ elements. When $F$ has enough $n$-th roots of unity and $F ¥ \mathbb{C}$, the Hilbert symbol provides a nondegenerate skew-symmetric bilinear map

$$
(\cdot, \cdot)_{F, n}: \frac{F^{\times}}{F^{\times n}} \otimes_{\mathbb{Z}} \frac{F^{\times}}{F^{\times n}} \rightarrow \mu_{n}(F) .
$$

In general, the Hilbert symbol is not alternating. The Hilbert symbol factors through $\boldsymbol{K}_{2}(F)$ via the universal symbol.

The definition of the Hilbert symbol relies on a choice of reciprocity isomorphism in local class field theory - this choice has been made earlier in sending a uniformizing element of $F^{\times}$to a geometric Frobenius.

If $F$ is nonarchimedean and $(p, n)=1$, then we say that the Hilbert symbol $(\cdot, \cdot)_{F, n}$ is tame. If $p$ is odd, then in the tame case, $(\varpi, \varpi)_{F, n}=(-1)^{(q-1) / n}$ for every uniformizing element $\varpi \in F^{\times}$. When $p=2$, in the tame case, $(\varpi, \varpi)_{F, n}=1$. When $F \cong \mathbb{R}$, we have $(-1,-1)_{F, 2}=-1$. 
Tori. Henceforth $\boldsymbol{T}$ will always denote an algebraic torus over $F$. Let $L$ be a finite Galois extension of $F$, over which $\boldsymbol{T}$ splits, and define $\Gamma=\operatorname{Gal}(L / F)$. We write $X=\operatorname{Hom}\left(\boldsymbol{T}, \boldsymbol{G}_{m}\right)$ for the character group and $Y$ for the cocharacter group $\operatorname{Hom}\left(\boldsymbol{G}_{m}, \boldsymbol{T}\right)$. We view $X$ and $Y$ as finite rank free $\mathbb{Z}$-modules endowed with actions of $\Gamma$. The groups $X$ and $Y$ are in canonical $\Gamma$-invariant duality.

The dual torus $\hat{\boldsymbol{T}}$ is the split torus $\operatorname{Spec}(\mathbb{Z}[Y])$ over $\mathbb{Z}$, with the resulting action of $\Gamma$. We write $\hat{\mathscr{T}}=\hat{\boldsymbol{T}}(\mathbb{C}) \equiv X \otimes_{\mathbb{Z}} \mathbb{C}^{\times}$for the resulting $\mathbb{C}$-torus, also endowed with the action of $\Gamma$.

Central extensions of tori by $\boldsymbol{K}_{2}$. Let $\mathfrak{C} \mathfrak{E x t}\left(\boldsymbol{T}, \boldsymbol{K}_{2}\right)$ be the category of central extensions of $\boldsymbol{T}$ by $\boldsymbol{K}_{2}$ in $\mathfrak{G p}_{F}$. Let $\mathfrak{C E r t}_{\Gamma}\left(Y, L^{\times}\right)$be the category of $\Gamma$-equivariant extensions of $Y$ by $L^{\times}$.

In [2001], Deligne and Brylinski study a category we will call $\mathfrak{D} \mathfrak{B}_{T}$. Its objects are pairs $(Q, \tilde{Y})$, where

- $Q: Y \rightarrow \mathbb{Z}$ is a $\Gamma$-invariant quadratic form;

- $\tilde{Y}$ is a $\Gamma$-equivariant central extension of $Y$ by $L^{\times}$; and

- the resulting commutator map $C: \bigwedge^{2} Y \rightarrow L^{\times}$satisfies

$$
C\left(y_{1}, y_{2}\right)=(-1)^{B_{Q}\left(y_{1}, y_{2}\right)} \quad \text { for all } y_{1}, y_{2} \in Y,
$$

where $B_{Q}$ is the symmetric bilinear form associated to $Q$.

If $\left(Q_{1}, \tilde{Y}_{1}\right)$, and $\left(Q_{2}, \tilde{Y}_{2}\right)$ are two objects of $\mathfrak{D} \mathfrak{B}_{T}$, then a morphism from $\left(Q_{1}, \tilde{Y}_{1}\right)$ to $\left(Q_{2}, \tilde{Y}_{2}\right)$ exists only if $Q_{1}=Q_{2}$, in which case the morphisms of $\mathfrak{D} \mathfrak{B}_{T}$ are the just those from $\tilde{Y}_{1}$ to $\tilde{Y}_{2}$ in $\mathfrak{C E x t}_{\Gamma}\left(Y, L^{\times}\right)$.

In [2001, Section 3.10], Deligne and Brylinski go on to construct an equivalence of categories from $\mathfrak{C} \mathfrak{E x t}\left(\boldsymbol{T}, \boldsymbol{K}_{2}\right)$ to the category $\mathfrak{D} \mathfrak{B}_{\boldsymbol{T}}$. In particular, given a central extension $\boldsymbol{T}^{\prime}$ of $\boldsymbol{T}$ by $\boldsymbol{K}_{2}$, their work (in part following [Esnault et al. 1998]) yields a quadratic form $Q: Y \rightarrow \mathbb{Z}$, and a central extension $\tilde{Y}$ of $Y$ by $L^{\times}$. Considering the central extension $\boldsymbol{T}^{\prime}(L)$ in

$$
1 \rightarrow K_{2}(L) \rightarrow \boldsymbol{T}^{\prime}(L) \rightarrow \boldsymbol{T}(L) \rightarrow 1,
$$

they show that the resulting commutator $C_{L}: \bigwedge^{2} \boldsymbol{T}(L) \rightarrow \boldsymbol{K}_{2}(L)$ satisfies

$$
C_{L}\left(y_{1}\left(l_{1}\right), y_{2}\left(l_{2}\right)\right)=\left\{l_{1}, l_{2}\right\}^{B_{Q}\left(y_{1}, y_{2}\right)} \quad \text { for all } y_{1}, y_{2} \in Y \text { and } l_{1}, l_{2} \in L^{\times} .
$$

Locally compact abelian groups. An LCA group is a locally compact Hausdorff separable abelian topological group. We work here in the category $\mathfrak{L} \mathfrak{C} \mathfrak{A} \mathfrak{b}$ whose objects are LCA groups and whose morphisms are continuous homomorphisms. Suppose that we are have a short exact sequence of LCA groups and continuous homomorphisms given by

$$
0 \rightarrow A \rightarrow B \rightarrow C \rightarrow 0 .
$$


Given a fourth LCA group $D$, the functor $\operatorname{Hom}(\bullet, D)$ is left-exact, yielding an exact sequence

$$
0 \rightarrow \operatorname{Hom}(C, D) \rightarrow \operatorname{Hom}(B, D) \rightarrow \operatorname{Hom}(A, D) .
$$

2.0.1. Continuous characters. When $A$ is an LCA group, we write $\mathscr{X}(A)$ for the group of continuous homomorphisms from $A$ to the LCA group $\mathbb{C}^{\times}$, under pointwise multiplication. We call elements of $\mathscr{X}(A)$ characters (or continuous characters) of $A$. If $\chi \in \mathscr{X}(A)$ and $|\chi(a)|=1$ for all $a \in A$, then we say that $\chi$ is a unitary character. We write $\hat{A}$ for the Pontryagin dual of $A$, that is, the set of unitary characters of $A$, with its natural topology as an LCA group.

We say that $A$ is an elementary LCA group if $A \cong \mathbb{R}^{a} \times \mathbb{Z}^{b} \times(\mathbb{R} / \mathbb{Z})^{c} \times F$ for some finite group $F$ and some nonnegative integers $a, b, c$. When $A$ is elementary, $\mathscr{L}(A)$ has a natural structure as a complex algebraic group. In the case above, $\mathscr{L}(A) \cong \mathbb{C}^{a} \times\left(\mathbb{C}^{\times}\right)^{b} \times \mathbb{Z}^{c} \times \hat{F}$.

If $A$ is generated by a compact neighborhood of the identity, then $A$ is canonically isomorphic to the inverse limit of its elementary quotients by compact subgroups. In this case, $\mathscr{X}(A)$ is endowed with the (inductive limit) structure of a complex algebraic group. In this paper, all LCA groups will be generated by a compact neighborhood of the identity, and thus $\mathscr{X}(A)$ will be viewed as a complex algebraic group.

2.0.2. Exactness criteria. Given a short exact sequence

$$
0 \rightarrow A \rightarrow B \rightarrow C \rightarrow 0
$$

there are two important cases in which the induced map $\mathscr{X}(B) \rightarrow \mathscr{L}(A)$ is surjective, leading to an exact sequence

$$
0 \rightarrow \mathscr{X}(C) \rightarrow \mathscr{L}(B) \rightarrow \mathscr{L}(A) \rightarrow 0 .
$$

Proposition 2.1. Suppose that $A$ is compact. Then $\mathscr{L}(B) \rightarrow \mathscr{X}(A)$ is surjective.

Proof. If $A$ is compact, every continuous character of $A$ is unitary. The exactness of Pontryagin duality implies that every unitary character of $A$ extends to a unitary character of $B$. Hence $\mathscr{L}(B)$ surjects onto $\mathscr{X}(A)$.

Proposition 2.2. Suppose that the map from $A$ to $B$ is an open embedding. Then $\mathscr{L}(B) \rightarrow \mathscr{L}(A)$ is surjective.

Proof. The proof, which is not difficult, follows directly from [Hoffmann and Spitzweck 2007, Proposition 3.3], for example. 
Complex varieties and groups. We use a script letter, such as $\mathcal{M}$, to denote the (complex) points of a complex algebraic variety. It is unnecessary for us to distinguish between complex varieties and their complex points. If $R$ is a commutative reduced finitely-generated $\mathbb{C}$-algebra, then we write $\mathcal{M}=\mathcal{M}(R)$ for the maximal ideal spectrum of $A$, viewed as a complex variety. We view $\mathbb{C}^{\times}$as a complex algebraic variety, identifying $\mathbb{C}^{\times} \equiv M \mathscr{Y}(\mathbb{C}[\mathbb{Z}])$, where $\mathbb{C}[\mathbb{Z}]$ denotes the group ring. We view $\mathbb{C}$ itself as an algebraic variety (the affine line over the field $\mathbb{C}$ ).

Let $\mathscr{G}$ be a complex algebraic group, or in other words, a group in the category of complex algebraic varieties. A $\mathscr{G}$-variety is a complex algebraic variety $M$ endowed with an action $\mathscr{G} \times \mathcal{M} \rightarrow \mathcal{M}$ that is complex-algebraic. A $\mathscr{G}_{\text {-torsor }}$ is a $\mathscr{G}$-variety $\mathcal{M}$ such that the induced map $\mathscr{G} \times \mathcal{M} \rightarrow \mathcal{M} \times \mathcal{M}$ sending $(g, m)$ to $(g \cdot m, m)$ is an isomorphism of complex algebraic varieties.

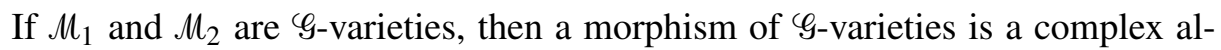
gebraic map from $\mu_{1}$ to $\mu_{2}$ that intertwines the action of $\mathscr{G}$. Morphisms of torsors are defined in the same way.

\section{Genuine representations of metaplectic tori}

In this section, we fix notation as follows:

- $F$ will be a local field, with $F ¥ \mathbb{C}$, and $n$ will be a positive integer such that $F$ has enough $n$-th roots of unity.

- $\boldsymbol{T}$ will be a torus over a local field $F$ which splits over a finite Galois extension $L / F$, with $\Gamma=\operatorname{Gal}(L / F) . X$ and $Y$ will be the resulting character and cocharacter groups.

- $\boldsymbol{T}^{\prime}$ will be an extension of $\boldsymbol{T}$ by $\boldsymbol{K}_{2}$ in $\mathfrak{G}_{F}$.

- $(Q, \tilde{Y})$ will be the Deligne-Brylinski invariants of $\boldsymbol{T}^{\prime} . B$ will be the symmetric bilinear form associated to $Q$.

- $\epsilon: \boldsymbol{\mu}_{n}(F) \rightarrow \mathbb{C}^{\times}$will be a fixed injective character.

Heisenberg groups. Suppose that $S$ is an LCA group and $A$ is a finite cyclic abelian group endowed with a faithful unitary character $\epsilon: A \rightarrow \mathbb{C}^{\times}$. Suppose that $\tilde{S}$ is a locally compact group that is a central extension of $S$ by $A$ (in the category of locally compact groups and continuous homomorphisms:

$$
1 \rightarrow A \rightarrow \tilde{S} \rightarrow S \rightarrow 1
$$

In this situation, the commutator on $\tilde{S}$ descends to a unique alternating form

$$
C: \bigwedge^{2} S \rightarrow A
$$


Let $Z(\tilde{S})$ be the center of $\tilde{S}$. Then $Z(\tilde{S})$ is the preimage of a subgroup $Z^{\dagger}(S) \subset S$, where $Z^{\dagger}(S)=\left\{s \in S\right.$ such that $C\left(s, s^{\prime}\right)=1$ for all $\left.s^{\prime} \in S\right\}$. Throughout this paper, the following condition will be satisfied, and hence we assume that

$Z^{\dagger}(S)$ is an open subgroup of finite index in $S$.

We define two sets:

- The set $\mathscr{L}_{\epsilon}(\tilde{S})$ of continuous genuine characters of $\tilde{S}$. These are elements of $\mathscr{X}(\tilde{S})$ whose restriction to $A$ equals $\epsilon$.

- The set $\Phi_{\epsilon}(\tilde{S})$ of irreducible genuine representations of $\tilde{S}$. These are irreducible (algebraic) representations $(\pi, V)$ of $\tilde{S}$ on a complex vector space, on which $Z(\tilde{S})$ acts via a continuous genuine character. In particular, since $Z(\tilde{S})$ will always have finite index in $\tilde{S}$, these are finite-dimensional representations.

We often use the following analogue of the Stone-von Neumann theorem:

Theorem 3.1. Suppose that $\chi \in \mathscr{X}_{\epsilon}(Z(\tilde{S}))$ is a genuine continuous character. Let $\tilde{M}$ denote a maximal commutative subgroup of $\tilde{S}$. Then there exists an extension $\tilde{\chi} \in \mathscr{L}(\tilde{M})$ of $\chi$ to $\tilde{M}$. Define a representation of $\tilde{S}$ by

$$
\left(\pi_{\chi}, V_{\chi}\right)=\operatorname{Ind}_{\tilde{M}}^{\tilde{S}} \tilde{\chi}
$$

Algebraic induction suffices here, since we always assume that $Z(\tilde{S})$ has finite index in $\tilde{S}$. Then

(1) the representation $\left(\pi_{\chi}, V_{\chi}\right)$ is irreducible;

(2) the representation $\left(\pi_{\chi}, V_{\chi}\right)$ has central character $\chi$;

(3) the isomorphism class of $\left(\pi_{\chi}, V_{\chi}\right)$ depends only upon $\chi$ and not upon the choices of subgroup $\tilde{M}$ and extension $\tilde{\chi}$;

(4) every irreducible representation of $\tilde{S}$ on which $Z(\tilde{S})$ acts via $\chi$ is isomorphic to $\left(\pi_{\chi}, V_{\chi}\right)$.

Proof. Extension of $\chi$ to $\tilde{M}$ follows from Proposition 2.2. All but the last claim are proved in [Kazhdan and Patterson 1984, Section 0.3] and follow directly from Mackey theory. The last claim follows from the previous claims and Frobenius reciprocity.

Metaplectic tori over local fields. The central extension of $\boldsymbol{T}$ by $\boldsymbol{K}_{2}$ yields a central extension of groups given by

$$
1 \rightarrow \boldsymbol{K}_{2}(F) \rightarrow \boldsymbol{T}^{\prime}(F) \rightarrow \boldsymbol{T}(F) \rightarrow 1 .
$$

Since $F$ is assumed to have enough $n$-th roots of unity, the Hilbert symbol allows us to push forward this extension to get

$$
1 \rightarrow \mu_{n} \rightarrow \tilde{T} \rightarrow T \rightarrow 1,
$$


where $\mu_{n}=\boldsymbol{\mu}_{n}(F)$ and $T=\boldsymbol{T}(F)$. By results of [Brylinski and Deligne 2001, Sections 10.2 and 10.3], which followed [Moore 1964], this is a topological central extension of the LCA group $T$ by the LCA group $\mu_{n}$.

In this case, the center $Z(\tilde{T})$ has finite index in $\tilde{T}$. Furthermore, Theorem 3.1 implies this:

Proposition 3.2. There is a natural bijection between the set $\Phi_{\epsilon}(\tilde{T})$ of irreducible genuine representations of $\tilde{T}$ and the set $\mathscr{X}_{\epsilon}(Z(\tilde{T}))$ of genuine characters of $Z(\tilde{T})$.

There is a short exact sequence $1 \rightarrow \mu_{n} \rightarrow Z(\tilde{T}) \rightarrow Z^{\dagger}(T) \rightarrow 1$ of LCA groups. Proposition 2.1 then implies:

Proposition 3.3. The space $\mathscr{X}_{\epsilon}(Z(\tilde{T}))$ of genuine continuous characters of $Z(\tilde{T})$ is a $\mathscr{L}\left(Z^{\dagger}(T)\right)$-torsor.

Corollary 3.4. The set $\Phi_{\epsilon}(\tilde{T})$ is a $\mathscr{L}\left(Z^{\dagger}(T)\right)$-torsor.

In particular, we give $\Phi_{\epsilon}(\tilde{T})$ the structure of a complex algebraic variety so that it is a complex algebraic $\mathscr{X}\left(Z^{\dagger}(T)\right)$-torsor.

Since $Z^{\dagger}(T)$ is a finite index subgroup of $T$, restriction of continuous characters yields a surjective homomorphism res: $\mathscr{L}(T) \rightarrow \mathscr{L}\left(Z^{\dagger}(T)\right)$ of complex algebraic groups. As a result, the set $\Phi_{\epsilon}(\tilde{T})$ is a homogeneous space for $\mathscr{X}(T)$, or equivalently (by Langlands's theorem [1997]), a homogeneous space for $H_{c}^{1}\left(\mathcal{W}_{L / F}, \hat{\mathcal{T}}\right)$.

\section{Split tori}

In this section, we keep the assumptions of the previous section. In addition, we assume that $\boldsymbol{T}$ is a split torus of rank $r$ over $F$. Thus, there is a canonical identification $\boldsymbol{T}(F) \equiv Y \otimes_{\mathbb{Z}} F^{\times}$. We are interested in parameterizing $\Phi_{\epsilon}(\tilde{T})$. By the results of the previous section, we may describe this set, up to a choice of base point, by describing the set $Z^{\dagger}(T)$.

An isogeny. Recall that $B: Y \otimes_{\mathbb{Z}} Y \rightarrow \mathbb{Z}$ is the symmetric bilinear form associated to $Q$. It allows us to construct a subgroup of finite index $Y^{\#} \subset Y$ by setting

$$
Y^{\#}=\left\{y \in Y \text { such that } B\left(y, y^{\prime}\right) \in n \mathbb{Z} \text { for all } y^{\prime} \in Y\right\} \text {. }
$$

Note that we suppress mention of $Q, B$, and $n$ in our notation $Y^{\#}$.

The subgroup $Y^{\#}$ can be related to the "Smith normal form" of the bilinear form $B$. Namely, there exists a pair of group isomorphisms $\alpha$ and $\beta$ with diagram

$$
\mathbb{Z}^{r} \stackrel{\beta}{\longleftarrow} Y \stackrel{\alpha}{\longrightarrow} \mathbb{Z}^{r}
$$

such that one has $B_{Q}\left(y_{1}, y_{2}\right)=D\left(\alpha\left(y_{1}\right), \beta\left(y_{2}\right)\right)$ for all $y_{1}, y_{2} \in Y$, and $D$ is a symmetric bilinear form on $\mathbb{Z}^{r}$ represented by a diagonal matrix with entries 
$\left(d_{1}, \ldots, d_{r}\right)$ (the elementary divisors). Let $e_{j}$ denote the smallest positive integer such that $d_{j} e_{j} \in n \mathbb{Z}$ for every $1 \leq j \leq r$. Then we find that

$$
Y^{\#}=\alpha^{-1}\left(e_{1} \mathbb{Z} \oplus e_{2} \mathbb{Z} \oplus \cdots \oplus e_{r} \mathbb{Z}\right) .
$$

Let $l: Y^{\#} \rightarrow Y$ denote the inclusion of $\mathbb{Z}$-modules. Since $Y^{\#}$ has finite index in $Y$, this corresponds to an $F$-isogeny $\iota: \boldsymbol{T}^{\#} \rightarrow \boldsymbol{T}$ of split tori, where $\boldsymbol{T}^{\#}$ is the split algebraic torus with cocharacter lattice $Y^{\#}$. From the previous observations, we find that

$$
\imath\left(T^{\#}\right)=\imath\left(\boldsymbol{T}^{\#}(F)\right)=\alpha^{-1}\left(F^{\times e_{1}} \times \cdots \times F^{\times e_{r}}\right) .
$$

Describing the center. Recall that extension $\tilde{T}$ of $T$ by $\mu_{n}$ yields a commutator $C: \bigwedge^{2} T \rightarrow \mu_{n}$. This commutator can be directly related to the bilinear form $B$; see [Brylinski and Deligne 2001]. If $u_{1}, u_{2} \in F^{\times}$and $y_{1}, y_{2} \in Y$, then one may directly compute

$$
C\left(y_{1}\left(u_{1}\right), y_{2}\left(u_{2}\right)\right)=\left(u_{1}, u_{2}\right)_{n}^{B\left(y_{1}, y_{2}\right)} .
$$

The diagonalization of $B$ via group isomorphisms $\alpha, \beta$ yields two isomorphisms of $F$-tori, given by

$$
\boldsymbol{G}_{m}^{r} \stackrel{\beta}{\longleftarrow} \boldsymbol{T} \stackrel{\alpha}{\longrightarrow} \boldsymbol{G}_{m}^{r} .
$$

One arrives at a bilinear form on $\left(F^{\times}\right)^{r}$, given by

$$
\Delta\left(\vec{z}_{1}, \vec{z}_{2}\right)=\prod_{j=1}^{r}\left(z_{1}^{(j)}, z_{2}^{(j)}\right)_{n}^{d_{j}} .
$$

This is related to the commutator $C$ by $C\left(t_{1}, t_{2}\right)=\Delta\left(\alpha\left(t_{1}\right), \beta\left(t_{2}\right)\right)$.

We can now characterize $Z^{\dagger}(T)$ :

Proposition 4.1. The subgroup $Z^{\dagger}(T)$ of $T$ is equal to the image of the isogeny $l$ on the $F$-rational points, that is, $Z^{\dagger}(T)=\imath\left(T^{\#}\right)$.

Proof. We find that

$$
\begin{array}{rlrl}
t_{1} \in Z^{\dagger}(T) \text { if and only if } & C\left(t_{1}, t_{2}\right) & =1 \text { for all } t_{2} \in T \\
& \text { if and only if } & \Delta\left(\alpha\left(t_{1}\right), \beta\left(t_{2}\right)\right) & =1 \text { for all } t_{2} \in T \\
& \text { if and only if } & \Delta\left(\alpha\left(t_{1}\right), \vec{z}_{2}\right) & =1 \text { for all } \vec{z}_{2} \in\left(F^{\times}\right)^{r} \\
& \text { if and only if } & \alpha\left(t_{1}\right) & \in\left(F^{\times e_{1}} \times \cdots \times F^{\times e_{r}}\right) \\
& \text { if and only if } & t_{1} & \in l\left(T^{\#}\right) .
\end{array}
$$

The penultimate step follows from the nondegeneracy of the Hilbert symbol.

We have now proved this:

Theorem 4.2. If $F$ is a local field, then $\Phi_{\epsilon}(\tilde{T})$ is a torsor for $\mathscr{X}\left({ }_{l}\left(T^{\#}\right)\right)$. 
Character groups. The previous theorem motivates the further analysis of the group $\mathscr{X}\left(\imath\left(T^{\#}\right)\right)$. We write $\imath^{*}$ for the pullback homomorphism $\imath^{*}: \mathscr{X}(T) \rightarrow \mathscr{L}\left(T^{\#}\right)$.

Proposition 4.3. There is a natural identification $\mathscr{X}\left(l\left(T^{\#}\right)\right) \equiv \operatorname{Im}\left(l^{*}\right)$.

Proof. There are short exact sequences

$$
1 \rightarrow \operatorname{ker}(\imath) \rightarrow T^{\#} \rightarrow \iota\left(T^{\#}\right) \rightarrow 1 \quad \text { and } \quad 1 \rightarrow \imath\left(T^{\#}\right) \rightarrow T \rightarrow \operatorname{cok}(\iota) \rightarrow 1
$$

of LCA groups. Using Propositions 2.1 and 2.2, we arrive at short exact sequences

$$
\begin{aligned}
& 1 \rightarrow \mathscr{X}\left(l\left(T^{\#}\right) \rightarrow \mathscr{X}\left(T^{\#}\right) \rightarrow \mathscr{X}(\operatorname{ker}(l)) \rightarrow 1,\right. \\
& 1 \rightarrow \mathscr{X}(\operatorname{cok}(l)) \rightarrow \mathscr{X}(T) \rightarrow \mathscr{X}\left(l\left(T^{\#}\right)\right) \rightarrow 1
\end{aligned}
$$

of character groups. Since $\mathscr{X}(T)$ surjects onto $\mathscr{X}\left(l\left(T^{\#}\right)\right)$, we find that the image of $\iota^{*}: \mathscr{X}(T) \rightarrow \mathscr{X}\left(T^{\#}\right)$ equals the image of the injective map $\mathscr{L}\left(\imath\left(T^{\#}\right)\right) \rightarrow \mathscr{X}\left(T^{\#}\right)$.

The dual complex. The isogeny of split $F$-tori $\iota: \boldsymbol{T}^{\#} \rightarrow \boldsymbol{T}$ yields an isogeny $\hat{\imath}: \hat{\mathscr{T}} \rightarrow \hat{\mathscr{T}}^{\#}$ of the complex dual tori. One may pull back continuous characters via $\imath^{*}: \mathscr{X}(T) \rightarrow \mathscr{L}\left(T^{\#}\right)$.

The following result follows from local class field theory, and demonstrates the naturality of Langlands's classification [1997].

Proposition 4.4. There is a commutative diagram

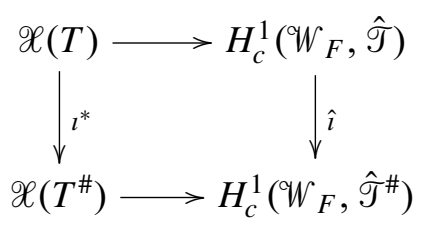

of complex algebraic groups, whose rows are the reciprocity isomorphisms of local class field theory.

Note that since $\boldsymbol{T}$ and $\boldsymbol{T}^{\#}$ are split tori, the continuous cohomology groups are simply given by $H_{c}^{1}\left(W_{F}, \hat{\mathfrak{T}}\right)=\operatorname{Hom}_{c}\left(W_{F}, \hat{\mathfrak{T}}\right)$.

Corollary 4.5. There is a natural identification

$$
\mathscr{L}\left(Z^{\dagger}(T)\right) \equiv \operatorname{Im}\left(\hat{\imath}: H_{c}^{1}\left(\mathcal{W}_{F}, \hat{\mathscr{T}}\right) \rightarrow H_{c}^{1}\left(\mathcal{W}_{F}, \hat{\mathscr{T}}^{\#}\right)\right) .
$$

Parameterization by hypercohomology. We may now parameterize representations using the hypercohomology of the complex of tori $\hat{\mathfrak{T}} \stackrel{\hat{\imath}}{\longrightarrow} \hat{\mathfrak{T}}^{\#}$ concentrated in degrees zero and one. We follow the treatment in the appendices of [Kottwitz and Shelstad 1999] when discussing continuous hypercohomology of Weil groups with coefficients in complexes of tori. In particular, we concentrate the complexes in degrees 0 and 1 following [Kottwitz and Shelstad 1999], and not in degrees -1 and 0 as in [Borovoi 1998]. 
There is a long exact sequence in cohomology that includes

$$
H_{c}^{1}\left(\mathcal{W}_{F}, \hat{\mathscr{T}} \rightarrow \hat{\mathscr{T}}^{\#}\right) \stackrel{\eta}{\longrightarrow} H_{c}^{1}\left(\mathcal{W}_{F}, \hat{\mathscr{T}}\right) \longrightarrow H_{c}^{1}\left(\mathcal{W}_{F}, \hat{\mathscr{T}}^{\#}\right) .
$$

Lemma 4.6. The homomorphism $\eta$ is injective.

Proof. Extending the long exact sequence above, it suffices to prove the surjectivity of the preceding homomorphism $H_{c}^{0}\left(\mathcal{W}_{F}, \hat{\mathscr{T}}\right) \rightarrow H_{c}^{0}\left(\mathcal{W}_{F}, \hat{\mathscr{T}}^{\#}\right)$. But $\hat{\mathscr{T}}$ and $\hat{\mathscr{T}}^{\#}$ are complex tori, trivial as $\mathcal{W}_{F}$-modules, and $\hat{\imath}$ is an isogeny. Therefore, the map above is surjective.

From this lemma, we identify the hypercohomology group $H_{c}^{1}\left(\mathcal{W}_{F}, \hat{\mathscr{T}} \rightarrow \hat{\mathscr{T}}^{\#}\right)$ with a subgroup of $H_{c}^{1}\left(\mathcal{W}_{F}, \hat{\mathscr{T}}\right)$.

Lemma 4.7. The group $H_{c}^{1}\left(\mathcal{W}_{F}, \hat{\mathscr{T}} \rightarrow \hat{\mathscr{T}}^{\#}\right)$ is finite.

Proof. Since $\hat{\imath}$ is an isogeny, it has finite kernel and cokernel. The lemma follows because there is a long exact sequence that includes the terms

$$
H_{c}^{1}\left(\mathcal{W}_{F}, \operatorname{ker}(\hat{\imath})\right) \rightarrow H_{c}^{1}\left(\mathcal{W}_{F}, \hat{\mathscr{T}} \rightarrow \hat{\mathscr{T}}^{\#}\right) \rightarrow H_{c}^{0}\left(W_{F}, \operatorname{cok}(\hat{\imath})\right) .
$$

This leads to the first main theorem:

Theorem 4.8. There exists an isomorphism

$$
\Im_{\epsilon}(\tilde{T}) \cong H_{c}^{1}\left(\mathcal{W}_{F}, \hat{\mathscr{T}}\right) / H_{c}^{1}\left(W_{F}, \hat{\mathscr{T}} \rightarrow \hat{\mathscr{T}}^{\#}\right)
$$

in the category of varieties over $\mathbb{C}$ endowed with an action of $H_{c}^{1}\left(\mathcal{W}_{F}, \hat{\mathcal{T}}\right)$.

Remark 4.9. The global analogue of this result also seems to hold. Let $\oiint_{\epsilon}^{\text {aut }}\left(\tilde{T}_{\AA}\right)$ denote the appropriate set of genuine automorphic representations of $\tilde{T}_{\mathbb{A}}$; it seems likely that

$$
و_{\epsilon}^{\text {aut }}\left(\tilde{T}_{\AA}\right) \cong H_{c}^{1}\left(\mathcal{W}_{F}, \hat{\mathfrak{T}}\right) / H_{c}^{1}\left(\mathcal{W}_{F}, \hat{\mathfrak{T}} \rightarrow \hat{\mathfrak{T}}^{\#}\right)
$$

when $\boldsymbol{T}$ is a split torus over a global field $F$. The proof follows the same techniques (together with the Hasse principle for isogenies of split tori), but requires some analytic care with extension of continuous characters and the appropriate Stonevon Neumann theorem. We hope to treat this global theorem in a future paper.

\section{Unramified tori}

For split tori, the cohomology groups that arise in Theorem 4.8 are quite simple, since $W_{F}$ acts trivially on $\hat{\mathscr{T}}$ and $\hat{\mathscr{T}}^{\#}$. In fact, the statement of this theorem makes sense even when $\boldsymbol{T}$ is a nonsplit torus. However, for general nonsplit tori, it seems that our explicit methods are insufficient to prove such a result. For "tame covers" of unramified tori over local nonarchimedean fields, such a paramaterization is possible.

In this section, we fix these notations: 
- $\boldsymbol{T}$ will be a local nonarchimedean field $F$, which splits over a finite unramified Galois extension $L / F$, with $\Gamma=\operatorname{Gal}(L / F) . X$ and $Y$ will be the resulting character and cocharacter groups.

- We define $d=[L: F]$ and write $\mathbf{I}$ for the residue field of $O_{L}$ (note that $\mathbf{I}$ has cardinality $q^{d}$ ).

- We fix a uniformizer $\varpi$ of $F^{\times}$(and hence of $L^{\times}$as well).

- We let $\gamma$ be the generator of $\Gamma$ that acts upon $\mathbf{I}$ via $\gamma(x)=x^{q}$. We let $r=\left(q^{d}-1\right) /(q-1)=\#\left(\mathbf{l}^{\times} / \mathbf{f}^{\times}\right)$.

- $\boldsymbol{T}^{\prime}$ will be an extension of $\boldsymbol{T}$ by $\boldsymbol{K}_{2}$ in $\mathfrak{G}_{F}$.

- $(Q, \tilde{Y})$ will be the Deligne-Brylinski invariants of $\boldsymbol{T}^{\prime} . B$ will be the symmetric bilinear form associated to $Q$.

- $n$ will be a positive integer such that $F$ has enough $n$-th roots of unity. We also assume that $(p, n)=1$.

- $\epsilon: \boldsymbol{\mu}_{n}(F) \rightarrow \mathbb{C}^{\times}$will be a fixed injective character.

- If $W$ is a subgroup of $Y$, then we write $W^{\Gamma}$ for the subgroup of $\Gamma$-fixed elements of $W$. We also define

$$
W^{\#}=\{y \in Y \text { such that } B(y, w) \in n \mathbb{Z} \text { for all } w \in W\} .
$$

$\mathbb{Z}[\Gamma]$-modules. $\Gamma$ is a cyclic group generated by $\gamma$ and of order $d$. Let $\mathbb{Z}[\Gamma]$ denote the integral group ring of $\Gamma$. We define the following elements of $\mathbb{Z}[\Gamma]$ :

- Let $\operatorname{Tr}=\sum_{i=0}^{d-1} \gamma^{i}$, and let $\operatorname{Tr}_{q}=\sum_{i=0}^{d-1} q^{i} \gamma^{i}$.

- Let $\delta=\gamma-1$, and let $\delta_{q}=q \gamma-1$.

Note that $\operatorname{Tr} \circ \delta=0$ and $\operatorname{Tr}_{q} \circ \delta_{q}=q^{d}-1$. When $M$ is an $\mathbb{Z}[\Gamma]$-module, we let $\bar{M}=M /\left(q^{d}-1\right) M$. We write $M^{\Gamma}$ for the $\Gamma$-invariant $\mathbb{Z}$-submodule of $M$. Therefore,

$$
M^{\Gamma}=\{m \in M \text { such that } \delta m=0\} .
$$

We define

$$
\bar{M}^{\Gamma, q}=\left\{\bar{m} \in \bar{M} \text { such that } \delta_{q} \bar{m}=0\right\} .
$$

Proposition 5.1. Suppose that $M$ is an $\mathbb{Z}[\Gamma]$-module. Then

$$
\operatorname{Tr}(M) \subset M^{\Gamma} \text { and } \operatorname{Tr}_{q}(\bar{M}) \subset \bar{M}^{\Gamma, q} .
$$

Proof. The first inclusion is obvious. For the second inclusion, suppose that $\bar{m} \in \bar{M}$. Then $\delta_{q} \operatorname{Tr}_{q} \bar{m}=\operatorname{Tr}_{q} \delta_{q} \bar{m}=\left(q^{d}-1\right) \bar{m}=0$.

Proposition 5.2. Suppose that $M$ is a $\mathbb{Z}[\Gamma]$-module that is free as an $\mathbb{Z}$-module. Then $\delta_{q}$ and $\operatorname{Tr}_{q}$ act as injective endomorphisms of $M$, and

$$
\operatorname{Im}\left(\delta_{q}\right)=\left\{m \in M \text { such that } \operatorname{Tr}_{q} m \in\left(q^{d}-1\right) M\right\} .
$$


Proof. Since $M$ is free as an $\mathbb{Z}$-module, $\delta_{q} \circ \operatorname{Tr}_{q}=\operatorname{Tr}_{q} \circ \delta_{q}=q^{d}-1$ acts as an injective endomorphism of $M$. Hence $\delta_{q}$ and $\operatorname{Tr}_{q}$ must also act as injective endomorphisms of $M$, proving the first assertion.

Since $\operatorname{Tr}_{q} \circ \delta_{q}=q^{d}-1$, it follows that

$$
\operatorname{Im}\left(\delta_{q}\right) \subset\left\{m \in M \text { such that } \operatorname{Tr}_{q} m \in\left(q^{d}-1\right) M\right\} .
$$

In the other direction, if $\operatorname{Tr}_{q} m \in\left(q^{d}-1\right) M$, then $\operatorname{Tr}_{q} m=\operatorname{Tr}_{q} \delta_{q} m^{\prime}$, for some $m^{\prime} \in M$. Since $\operatorname{Tr}_{q}$ acts via an injective endomorphism, it follows that $m=\delta_{q} m^{\prime}$.

Unramified tori. Much of our treatment of unramified tori is inspired by Ono [1961, Section 2]. Recall that $X$ and $Y$ are naturally $\mathbb{Z}[\Gamma]$-modules, and the pairing is $\Gamma$-invariant.

We fix a smooth model $\underline{\boldsymbol{T}}$ of $\boldsymbol{T}$ over $\mathrm{O}_{F}$. We make the identifications

$$
T_{L}=\boldsymbol{T}(L) \equiv Y \otimes_{\mathbb{Z}} L^{\times} \quad \text { and } \quad T_{F}=\boldsymbol{T}(F) \equiv\left(Y \otimes_{\mathbb{Z}} L^{\times}\right)^{\Gamma} .
$$

Similarly, for the integral points, we identify

$$
T_{L}^{\circ}=\underline{\boldsymbol{T}}\left(\mathcal{O}_{L}\right) \equiv Y \otimes_{\mathbb{Z}} \mathcal{O}_{L}^{\times} \quad \text { and } \quad T_{F}^{\circ}=\underline{\boldsymbol{T}}\left(\mathscr{O}_{F}\right) \equiv\left(Y \otimes_{\mathbb{Z}} \mathcal{O}_{L}^{\times}\right)^{\Gamma} .
$$

We write $\overline{\boldsymbol{T}}$ for the special fibre of $\underline{\boldsymbol{T}}$. Then, we also identify

$$
\bar{T}_{\mathbf{l}}=\overline{\boldsymbol{T}}(\mathbf{l}) \equiv Y \otimes_{\mathbb{Z}} \mathbf{l}^{\times} \quad \text { and } \quad \bar{T}_{\mathbf{f}}=\overline{\boldsymbol{T}}(\mathbf{f}) \equiv\left(Y \otimes_{\mathbb{Z}} \mathbf{l}^{\times}\right)^{\Gamma} .
$$

There are natural reduction homomorphisms $T_{L}^{\circ} \rightarrow \bar{T}_{\mathbf{1}}$ and $T_{F}^{\circ} \rightarrow \bar{T}_{\mathbf{f}}$. Let $T_{L}^{1}$ and $T_{F}^{1}$ denote the kernels of these reduction maps. The reduction morphisms are split by the Teichmüller lift, and we arrive at a decomposition $T_{L}^{\circ} \equiv T_{L}^{1} \times \bar{T}_{1}$ of $\mathbb{Z}[\Gamma]$-modules. Together with the valuation map, we arrive at a short exact sequence $1 \rightarrow T_{L}^{1} \times \bar{T}_{\mathbf{1}} \rightarrow T_{L} \rightarrow Y \rightarrow 1$ of $\mathbb{Z}[\Gamma]$-modules. The choice of ( $\Gamma$-invariant) uniformizing element $\varpi$ splits this exact sequence, leading to a decomposition of $\mathbb{Z}[\Gamma]$-modules given by $T_{L} \equiv Y \times \bar{T}_{1} \times T_{L}^{1}$.

We use this decomposition to "get our hands on" elements of $T_{L}$. First, every element of $T_{L}$ can be expressed as $y(\varpi) t^{\circ}$ for uniquely determined $y \in Y$ and $t^{\circ} \in T_{L}^{\circ}$. Let $\theta_{\mathbf{l}}$ denote a generator of the cyclic group $\mathbf{l}^{\times}$, and let $\theta_{\mathbf{f}}=\theta_{\mathbf{l}}^{r}$. Thus $\theta_{\mathbf{f}}$ is a generator of the cyclic group $\mathbf{f}^{\times}$. Let $\vartheta_{L} \in \mathbb{O}_{L}^{\times}$and $\vartheta_{F} \in \mathbb{O}_{F}^{\times}$denote the Teichmüller lifts of $\theta_{\mathbf{l}}$ and $\theta_{\mathbf{f}}$, respectively.

Let $\zeta_{L}=\left(\varpi, \vartheta_{L}\right)_{L, q^{d}-1}$. Let $\zeta_{F}=\zeta_{L}^{r}$. Note that $\zeta_{L}$ is a primitive $\left(q^{d}-1\right)$-st root of unity, and $\zeta_{F}$ is a primitive $(q-1)$-st root of unity.

Recall that $\bar{Y}=Y /\left(q^{d}-1\right) Y$; thus, for $\bar{y} \in \bar{Y}$, it makes sense to write $\bar{y}\left(\vartheta_{L}\right)$ as an element of $T_{L}^{\circ}$. According to the decomposition $T_{L} \equiv Y \times \bar{T}_{\mathbf{I}} \times T_{L}^{1}$, every element $t \in T_{L}$ has a unique expression as $t=y_{1}(\varpi) \bar{y}_{2}\left(\vartheta_{L}\right) t^{1}$, where $y_{1} \in Y, \bar{y}_{2} \in \bar{Y}$, and $t^{1} \in T_{L}^{1}$. To determine when such an expression lies in $T_{F}$, we have the following characterization: 
Proposition 5.3. An element $y_{1}(\varpi) \bar{y}_{2}\left(\vartheta_{L}\right) t^{1}$ of $T_{L}$, with $y_{1}, \bar{y}_{2}$, and $t^{1}$ as above, lies in $T_{F}$ if and only if

- $y_{1} \in Y^{\Gamma}$, that is, $\delta\left(y_{1}\right)=0$;

- $\bar{y}_{2} \in \bar{Y}^{\Gamma, q}$, that is, $\delta_{q}\left(\bar{y}_{2}\right)=0$; and

- $t^{1} \in T_{F}^{1}$.

Proof. By the $\Gamma$-invariance of the decomposition $T_{L} \equiv Y \times \bar{T}_{\mathrm{I}} \times T_{L}^{1}$, we find that $y_{1}(\varpi) \bar{y}_{2}\left(\vartheta_{L}\right) t^{1} \in T_{F}$ if and only if the three factors are fixed by $\Gamma$. The proposition follows from three observations:

- Since $\varpi \in F$, we have $y_{1}(\varpi) \in T_{L}^{\Gamma}$ if and only if $y_{1} \in Y^{\Gamma}$.

- Since $\gamma\left(\vartheta_{L}\right)=\vartheta_{L}^{q}$, we find that $\bar{y}_{2}\left(\vartheta_{L}\right) \in T_{L}^{\Gamma}$ if and only if $\bar{y}_{2}=q \gamma\left(\bar{y}_{2}\right)$ in $\bar{Y}$.

- Since the reduction map intertwines the action of $\Gamma$, we have $t^{1} \in\left(T_{L}^{1}\right)^{\Gamma}$ if and only if $t^{1} \in T_{F}^{1}$.

Tame metaplectic unramified tori. The structure of $\boldsymbol{T}^{\prime}(L)$ and $\boldsymbol{T}^{\prime}(F)$ is based on [Brylinski and Deligne 2001, Sections 12.8-12.12]. In particular, if we let $T_{L}^{\prime}=$ $\boldsymbol{T}^{\prime}(L)$ and $T_{F}^{\prime}=\boldsymbol{T}^{\prime}(F)$, there is a natural commutative diagram

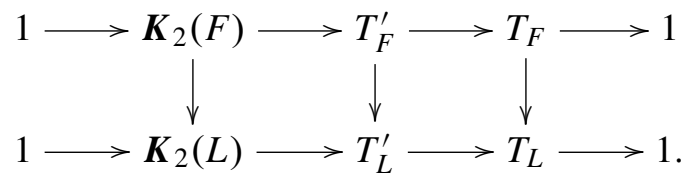

There is a natural action of $\Gamma$ on the bottom row such that $\boldsymbol{K}_{2}(F)$ maps to $\boldsymbol{K}_{2}(L)^{\Gamma}$, $T_{F}=T_{L}^{\Gamma}$, and $T_{F}^{\prime}$ maps to $\left(T_{L}^{\prime}\right)^{\Gamma}$. The tame symbols yield a commutative diagram

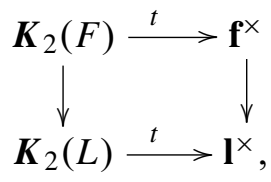

where the downward arrows arise from the functoriality of $\boldsymbol{K}_{2}$ and $\boldsymbol{K}_{1}$. The bottom row is a morphism of $\mathbb{Z}[\Gamma]$-modules. Pushing forward $T_{F}^{\prime}$ and $T_{L}^{\prime}$ via the tame symbols yields a commutative diagram of locally compact groups, with exact rows:

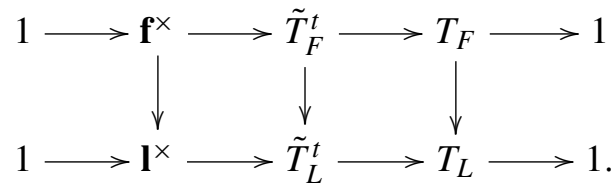

The downward arrows arise from the inclusion of $F$ in $L$, and of $\mathbf{f}$ in $\mathbf{l}$. Deligne and Brylinski, in [2001, Section 12.8], note the following: 
Proposition 5.4. In the commutative diagram (5-1), the groups in the top row are precisely the $\Gamma$-invariant subgroups of the bottom row. In other words, $\mathbf{f}^{\times}=\left(\mathbf{l}^{\times}\right)^{\Gamma}$, $T_{F}=T_{L}^{\Gamma}$, and $\tilde{T}_{F}^{t}=\left(\tilde{T}_{L}^{t}\right)^{\Gamma}$.

We may push forward the covers further to obtain all tame covers. Recall that $(p, n)=1$ and $F$ has enough $n$-th roots of unity. Then, we find $n$ divides $(q-1)$, and one obtains a natural surjective map

$$
\psi_{F}: \mathbf{f}^{\times} \rightarrow \boldsymbol{\mu}_{n}(F),
$$

by first applying the Teichmüller map (from $\mathbf{f}^{\times}$to $\boldsymbol{\mu}_{q-1}(F)$ ), and then raising to the power $m=(q-1) / n$. Recall that $r=\left(q^{d}-1\right) /(q-1)$. One gets a similar map

$$
\psi_{L}: \mathbf{l}^{\times} \rightarrow \boldsymbol{\mu}_{n r}(L)
$$

by applying the Teichmüller map (from $\mathbf{I}^{\times}$to $\boldsymbol{\mu}_{q^{d}-1}(L)$ ) and then raising to the power $m=(q-1) / n$. The compatibility of these maps yields a new commutative diagram with exact rows:

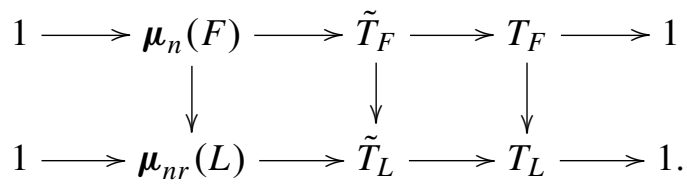

With this construction, we say that $\tilde{T}_{F}$ is a tame metaplectic cover of $T_{F}$, and $\tilde{T}_{L}$ is a tame metaplectic cover of $T_{L}$ as well. $\tilde{T}_{F}$ is identified as a subgroup of $\tilde{T}_{L}$.

Note that the commutator map for $\tilde{T}_{L}$ satisfies

$$
C_{L}\left(y_{1}(u), y_{2}(v)\right)=(u, v)_{L, n r}^{B\left(y_{1}, y_{2}\right)}=(u, v)_{L, q^{d}-1}^{m B\left(y_{1}, y_{2}\right)},
$$

where $(\cdot, \cdot)_{L, n r}$ and $(\cdot, \cdot)_{L, q^{d}-1}$ denote the appropriate Hilbert symbols (in this case, norm residue symbols) on $L^{\times}$. The commutator on $T_{F}$ is simply the restriction of $C_{L}$. As a result, $Z^{\dagger}\left(T_{F}\right) \supset Z^{\dagger}\left(T_{L}\right) \cap T_{F}$, where the preimage of $Z^{\dagger}\left(T_{F}\right)$ is the center of $\tilde{T}_{F}$ and the preimage of $Z^{\dagger}\left(T_{L}\right)$ is the center of $\tilde{T}_{L}$.

Computation of the center. We now recall that the set $\Phi_{\epsilon}\left(\tilde{T}_{F}\right)$ is a torsor for $\mathscr{L}\left(Z^{\dagger}\left(T_{F}\right)\right)$. Therefore we wish to study the group $Z^{\dagger}\left(T_{F}\right)$ in more detail. To this end, we first observe this:

Proposition 5.5. The group $T_{L}^{1}$ is contained in $Z^{\dagger}\left(T_{L}\right)$. Similarly, $T_{F}^{1}$ is contained in $Z^{\dagger}\left(T_{F}\right)$.

Proof. Since $\left(q^{d}-1, p\right)=1$, the Hilbert symbol (in this case, a norm-residue symbol) is trivial when one of its "inputs" is contained in $0_{L}^{1}$. Hence the commutator $C_{L}(\cdot, \cdot)$ is trivial when one of its inputs is contained in $T_{L}^{1}$. Hence $T_{L}^{1} \subset Z^{\dagger}\left(T_{L}\right)$. Since $Z^{\dagger}\left(T_{F}\right) \supset Z^{\dagger}\left(T_{L}\right) \cap T_{F}$, we find that $T_{F}^{1} \subset Z^{\dagger}\left(T_{F}\right)$ as well. 
Since $T_{F}^{1}$ is contained in $Z^{\dagger}\left(T_{F}\right), Z^{\dagger}\left(T_{F}\right)$ corresponds to a subgroup of $T_{F} / T_{F}^{1}$. Our choice of uniformizing element, together with the previously mentioned splittings, yields a decomposition $T_{L} / T_{L}^{1} \equiv Y \times \bar{T}_{1}$ of $\mathbb{Z}[\Gamma]$-modules. Namely, every element $t$ of $T_{L} / T_{L}^{1}$ can be represented by $y_{1}(\varpi) \bar{y}_{2}\left(\vartheta_{L}\right)$, for uniquely determined $y_{1} \in Y$ and $\bar{y}_{2} \in \bar{Y}$.

In order to describe $Z^{\dagger}\left(T_{F}\right)$, we work with a number of subgroups of $Y$. Recall that $Y^{\Gamma \#}=\left\{y \in Y\right.$ such that $B\left(y, y^{\prime}\right) \in n \mathbb{Z}$ for all $\left.y^{\prime} \in Y^{\Gamma}\right\}$. Note that $Y^{\Gamma \#} \supset Y^{\#}$. Also, it is important to distinguish between $Y^{\Gamma \#}$ and $Y^{\# \Gamma}=\left(Y^{\#}\right)^{\Gamma}$.

Lemma 5.6. There are inclusions of $\mathbb{Z}[\Gamma]$-modules, of finite index in $Y$, given by

$$
Y \supset Y^{\Gamma \#} \supset Y^{\#} \supset\left(q^{d}-1\right) Y .
$$

Furthermore, $\delta_{q}(Y) \subset Y^{\Gamma \#}$, and $\operatorname{Tr}_{q}\left(Y^{\Gamma \#}\right) \subset Y^{\#}$.

Proof. The inclusions are clear, since $n$ divides $q^{d}-1$. If $y \in Y$ and $y^{\prime} \in Y^{\Gamma}$, then we find

$$
\begin{aligned}
B\left(\delta_{q} y, y^{\prime}\right) & =B\left(q \gamma y-y, y^{\prime}\right) \\
& =q B\left(y, \gamma^{-1} y^{\prime}\right)-B\left(y, y^{\prime}\right) \\
& =(q-1) B\left(y, y^{\prime}\right) \in n \mathbb{Z} \quad(\text { since } q-1=m n) .
\end{aligned}
$$

Hence $\delta_{q}(Y) \subset Y^{\Gamma \#}$.

Now, suppose that $w \in Y^{\Gamma \#}$ and $y^{\prime} \in Y$. Then, we find

$$
\begin{aligned}
& B\left(\operatorname{Tr}_{q}(w), y^{\prime}\right)=\sum_{i=0}^{d-1} B\left(q^{i} \gamma^{i} w, y^{\prime}\right) \\
& \equiv \sum_{i=0}^{d-1} B\left(\gamma^{i} w, y^{\prime}\right)(\bmod n) \quad(\text { since } q-1=m n) \\
& \equiv B\left(w, \operatorname{Tr}\left(y^{\prime}\right)\right) \in n \mathbb{Z} \quad\left(\text { since } \operatorname{Tr}\left(y^{\prime}\right) \in Y^{\Gamma}\right) .
\end{aligned}
$$

Hence $\operatorname{Tr}_{q}\left(Y^{\Gamma \#}\right) \in Y^{\#}$.

Now, we fully describe $Z^{\dagger}\left(T_{F}\right)$ with two results:

Theorem 5.7. Let $y_{1} \in Y$ and $\bar{y}_{2} \in \bar{Y}$. Then if the element $t=y_{1}(\varpi) \bar{y}_{2}\left(\vartheta_{L}\right)$ is contained in $Z^{\dagger}\left(T_{F}\right)$, then for every lift $y_{2} \in Y$ of $\bar{y}_{2}$,

$$
y_{1}, y_{2} \in Y^{\#} \quad \text { and } \quad \delta_{q} y_{2} \in\left(q^{d}-1\right) Y^{\Gamma \#} .
$$

Proof. For reference during this proof, we recall that

$$
n m=q-1, \quad r=1+q+\cdots+q^{d-1}, \quad n m r=q^{d}-1 .
$$


Suppose furthermore that $y_{1}^{\prime}, y_{2}^{\prime} \in Y$, and let $\bar{y}_{2}^{\prime} \in \bar{Y}$ be the reduction of $y_{2}^{\prime}$. Then we find that $\left[\operatorname{Tr}\left(y_{1}^{\prime}\right)\right](\varpi)$ and $\left[\operatorname{Tr}_{q}\left(\bar{y}_{2}^{\prime}\right)\right]\left(\vartheta_{L}\right)$ are elements of $T_{F}$. It follows that $C_{L}\left(y_{1}(\varpi) \bar{y}_{2}\left(\vartheta_{L}\right),\left[\operatorname{Tr}\left(y_{1}^{\prime}\right)\right](\varpi)\right)=1$ and $C_{L}\left(y_{1}(\varpi) \bar{y}_{2}\left(\vartheta_{L}\right),\left[\operatorname{Tr}_{q}\left(\bar{y}_{2}^{\prime}\right)\right]\left(\vartheta_{L}\right)\right)=1$.

The explicit formula for the commutator $C_{L}$ yields

$$
\begin{aligned}
& 1=C_{L}\left(y_{1}(\varpi) y_{2}\left(\vartheta_{L}\right),\left[\operatorname{Tr}_{q}\left(\bar{y}_{2}^{\prime}\right)\right]\left(\vartheta_{L}\right)\right) \\
& =\prod_{i=0}^{d-1}\left(\varpi, \vartheta_{L}\right)_{L, q^{d}-1}^{m q^{i} B\left(y_{1}, \gamma^{i} y_{2}^{\prime}\right)} \\
& =\zeta_{L}^{\sum_{i=0}^{d-1} m q^{i} B\left(y_{1}, \gamma^{i} y_{2}^{\prime}\right)} \quad\left(\text { since }\left(\varpi, \vartheta_{L}\right)_{L, q^{d}-1}=\zeta_{L}\right) \\
& =\zeta_{L}^{\sum_{i=0}^{d-1} m q^{i} B\left(\gamma^{d-i} y_{1}, y_{2}^{\prime}\right)} \quad \text { (by the } \Gamma \text {-invariance of } B \text { ) }
\end{aligned}
$$

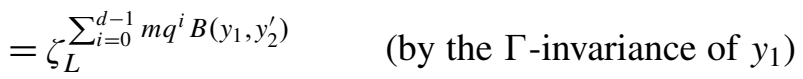

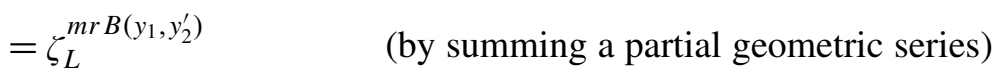

$$
\begin{aligned}
& =\zeta_{F}^{m B\left(y_{1}, y_{2}^{\prime}\right)} \quad\left(\text { since } \zeta_{F}=\zeta_{L}^{r}\right) \text {. }
\end{aligned}
$$

Since $1=\zeta_{F}^{m B\left(y_{1}, y_{2}^{\prime}\right)}$ for all $y_{2}^{\prime} \in Y$, we find that $y_{1} \in Y^{\#}$.

Carrying out a similar analysis, an explicit computation yields

$$
1=C_{L}\left(y_{1}(\varpi) y_{2}\left(\vartheta_{L}\right),\left[\operatorname{Tr}\left(y_{1}^{\prime}\right)\right](\varpi)\right)=\prod_{i=0}^{d-1}(\varpi, \varpi)_{L, q^{d}-1}^{m B\left(y_{1}, \gamma^{i} y_{1}^{\prime}\right)}\left(\varpi, \vartheta_{L}\right)_{L, q^{d}-1}^{m B\left(y_{2}, \gamma^{i} y_{1}^{\prime}\right)} .
$$

Now if $p$ is odd, we find that $q-1$ is even. Since $(\varpi, \varpi)_{L, q^{d}-1}= \pm 1$, and $m B\left(y_{1}, \gamma^{i} y_{1}^{\prime}\right) \in m n \mathbb{Z}=(q-1) \mathbb{Z} \subset 2 \mathbb{Z}$ (since $\left.y_{1} \in Y^{\#}\right)$, we find that

$$
(\varpi, \varpi)_{L, q^{d}-1}^{m B\left(y_{1}, \gamma^{i} y_{1}^{\prime}\right)}=1 .
$$

On the other hand, if $p=2$, then $(\varpi, \varpi)_{L, q^{d}-1}=1$, and once again the equality above holds. Continuing our computations yields

$$
\begin{aligned}
& 1=\prod_{i=0}^{d-1}(\varpi, \varpi)_{L, q^{d}-1}^{m B\left(y_{1}, \gamma^{i} y_{1}^{\prime}\right)}\left(\varpi, \vartheta_{L}\right)_{L, q^{d}-1}^{m B\left(y_{2}, \gamma^{i} y_{1}^{\prime}\right)} \\
& =\prod_{i=0}^{d-1} \zeta_{L}^{m B\left(y_{2}, \gamma^{i} y_{1}^{\prime}\right)} \quad\left(\text { since }(\varpi, \varpi)_{L, q^{d}-1}^{m B\left(y_{2}, \gamma^{i} y_{1}^{\prime}\right)}=1 \text { and }\left(\varpi, \vartheta_{L}\right)_{L, q^{d}-1}=\zeta_{L}\right) \\
& =\zeta_{L}^{\sum_{i=0}^{d-1} m q^{i} B\left(y_{2}, y_{1}^{\prime}\right)} \quad\left(\text { since } q \gamma\left(\bar{y}_{2}\right)=\bar{y}_{2}\right)
\end{aligned}
$$

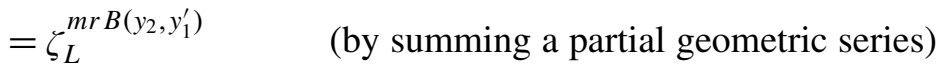

$$
\begin{aligned}
& =\zeta_{F}^{m B\left(y_{2}, y_{1}^{\prime}\right)} \quad\left(\text { since } \zeta_{F}=\zeta_{L}^{r}\right) \text {. }
\end{aligned}
$$


Hence, we find that $y_{2} \in Y^{\#}$.

Finally, we prove that $\delta_{q} y_{2} \in\left(q^{d}-1\right) Y^{\Gamma \#}$. Note that $\delta_{q} y_{2} \in\left(q^{d}-1\right) Y$ because $\bar{y}_{2} \in \bar{Y}^{\Gamma, q}$. Thus, $\delta_{q} y_{2}=\left(q^{d}-1\right) y_{3}$ for some $y_{3} \in Y$. It suffices to prove that $y_{3} \in Y^{\Gamma \#}$.

Now, to prove that $y_{3} \in Y^{\Gamma \#}$, suppose that $y^{\prime} \in Y^{\Gamma}$. It follows that

$$
\begin{aligned}
1 & =C_{L}\left(y_{1}(\varpi) \bar{y}_{2}\left(\vartheta_{L}\right), y^{\prime}(\varpi)\right) \\
& =(\varpi, \varpi)_{q^{d}-1}^{m B\left(y_{1}, y^{\prime}\right)}\left(\vartheta_{L}, \varpi\right)_{q^{d}-1}^{m B\left(y_{2}, y^{\prime}\right)}=\zeta_{L}^{m B\left(y_{2}, y^{\prime}\right)} .
\end{aligned}
$$

Hence $B\left(y_{2}, y^{\prime}\right) \in n r \mathbb{Z}$. It follows that

$$
\begin{aligned}
B\left(y_{3}, y^{\prime}\right) & =\left(q^{d}-1\right)^{-1} B\left(\delta_{q} y_{2}, y^{\prime}\right) \\
& =\left(q^{d}-1\right)^{-1}\left(B\left(q \gamma y_{2}, y^{\prime}\right)-B\left(y_{2}, y^{\prime}\right)\right)=r^{-1} B\left(y_{2}, y^{\prime}\right) \in n \mathbb{Z} .
\end{aligned}
$$

Thus $y_{3} \in Y^{\Gamma \#}$.

Theorem 5.8. Suppose that $y_{1}, y_{2} \in Y^{\#}$. Also suppose that $y_{1} \in Y^{\Gamma}$ and $\bar{y}_{2} \in \bar{Y}^{\Gamma, q}$. Finally, suppose that $\delta_{q} y_{2} \in\left(q^{d}-1\right) Y^{\Gamma \#}$. Then $y_{1}(\varpi) \bar{y}_{2}\left(\vartheta_{L}\right) \in Z^{\dagger}\left(T_{F}\right)$.

Proof. Since $y_{1} \in Y^{\Gamma}$ and $\bar{y}_{2} \in \bar{Y}^{\Gamma, q}$, it follows that $y_{1}(\varpi) \bar{y}_{2}\left(\vartheta_{L}\right) \in T_{F}$. Now we may compute some commutators.

Suppose that $y_{1}^{\prime} \in Y^{\Gamma}, \quad y_{2}^{\prime} \in Y$, and $\bar{y}_{2}^{\prime} \in \bar{Y}^{\Gamma, q}$. Thus $y_{1}^{\prime}(\varpi)$ and $\bar{y}_{2}^{\prime}\left(\vartheta_{L}\right)$ are elements of $T_{F}$. We begin by computing

$$
C_{L}\left(y_{1}(\varpi), y_{1}^{\prime}(\varpi)\right)=(\varpi, \varpi)_{L, q^{d}-1}^{m B\left(y_{1}, y_{1}^{\prime}\right)} .
$$

If $p$ is odd, then $m n=q-1$ is even, and thus $m B\left(y_{1}, y_{1}^{\prime}\right)$ is even. Hence the commutator is trivial. If $p$ is even, then $q^{d}-1$ is odd, and hence $(\varpi, \varpi)_{L, q^{d}-1}=1$. In either case, the commutator is trivial.

Now, consider the commutator $C_{L}\left(y_{1}(\varpi), \bar{y}_{2}^{\prime}\left(\vartheta_{L}\right)\right)=\zeta_{L}^{m B\left(y_{1}, y_{2}^{\prime}\right)}$. We claim that $m B\left(y_{1}, y_{2}^{\prime}\right) \in\left(q^{d}-1\right) \mathbb{Z}$. Indeed, we have

$$
B\left(y_{1}, y_{2}^{\prime}\right)=B\left(\gamma y_{1}, y_{2}^{\prime}\right)=B\left(y_{1}, \gamma^{-1} y_{2}^{\prime}\right)=B\left(y_{1}, q y_{2}^{\prime}+\left(q^{d}-1\right) y_{3}^{\prime}\right),
$$

for some $y_{3}^{\prime} \in Y$. Since $y_{1} \in Y^{\#}$, we have $B\left(y_{1},\left(q^{d}-1\right) y_{3}^{\prime}\right) \in n\left(q^{d}-1\right) \mathbb{Z}$. It follows that $(q-1) B\left(y_{1}, y_{2}^{\prime}\right) \in n\left(q^{d}-1\right) \mathbb{Z}$. From this, we find $B\left(y_{1}, y_{2}^{\prime}\right) \in n r \mathbb{Z}$. Hence $m B\left(y_{1}, y_{2}^{\prime}\right) \in m n r \mathbb{Z}=\left(q^{d}-1\right) \mathbb{Z}$. This proves our claim, and we have proved that $C_{L}\left(y_{1}(\varpi), \bar{y}_{2}^{\prime}\left(\vartheta_{L}\right)=1\right.$.

Next, consider the commutator $C_{L}\left(\bar{y}_{2}\left(\vartheta_{L}\right), y_{1}^{\prime}(\varpi)\right)=\zeta_{L}^{-m B\left(y_{2}, y_{1}^{\prime}\right)}$. We claim now that $m B\left(y_{2}, y_{1}^{\prime}\right) \in\left(q^{d}-1\right) \mathbb{Z}$. Indeed, we have

$$
B\left(y_{2}, y_{1}^{\prime}\right)=B\left(q \gamma y_{2}+\left(q^{d}-1\right) y_{3}, y_{1}^{\prime}\right)=q B\left(y_{2}, y_{1}^{\prime}\right)+\left(q^{d}-1\right) B\left(y_{3}, y_{1}^{\prime}\right)
$$


for some $y_{3} \in Y^{\Gamma \#}$. In particular, $B\left(y_{3}, y_{1}^{\prime}\right) \in n \mathbb{Z}$ since $y_{1}^{\prime} \in Y^{\Gamma}$. It follows that $(q-1) B\left(y_{2}, y_{1}^{\prime}\right) \in n\left(q^{d}-1\right) \mathbb{Z}$. From this we find that $B\left(y_{2}, y_{1}^{\prime}\right) \in n r \mathbb{Z}$, from which the claim follows. We have proved that $C_{L}\left(\bar{y}_{2}\left(\vartheta_{L}\right), y_{1}^{\prime}(\varpi)\right)=1$.

Finally, note that $\left(\vartheta_{L}, \vartheta_{L}\right)_{L, q^{d}-1}=1$. Hence $C_{L}\left(\bar{y}_{2}\left(\vartheta_{L}\right), \bar{y}_{2}^{\prime}\left(\vartheta_{L}\right)\right)=1$. We have proved that $y_{1}(\varpi)$ and $\bar{y}_{2}\left(\vartheta_{L}\right)$ commute with a set of generators for $T_{F} / T_{F}^{1}$. Since $T_{F}^{1} \in Z^{\dagger}\left(T_{F}\right)$, this suffices to prove that $y_{1}(\varpi) \bar{y}_{2}\left(\vartheta_{L}\right) \in Z^{\dagger}\left(T_{F}\right)$.

The previous two theorems fully characterize the subgroup $Z^{\dagger}\left(T_{F}\right)$.

Corollary 5.9. Suppose that $y_{1} \in Y, \bar{y}_{2} \in \bar{Y}$, and $t^{1} \in T_{F}^{1}$. Then $t=y_{1}(\varpi) \bar{y}_{2}\left(\vartheta_{L}\right) t^{1}$ belongs to $Z^{\dagger}\left(T_{F}\right)$ if and only if

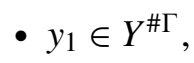

- $y_{2} \in Y^{\#}$ for any choice of representative $y_{2}$ of $\bar{y}_{2}$, and

- $\delta_{q} y_{2} \in\left(q^{d}-1\right) Y^{\Gamma \#}$ for any choice of representative $y_{2}$ of $\bar{y}_{2}$.

Proof. This corollary follows directly from the previous two theorems. One important observation is that the latter two conditions do not depend upon the choice of representative $y_{2} \in Y$ for a given $\bar{y}_{2} \in \bar{Y}$.

Indeed, suppose that $y_{2}^{\prime}=y_{2}+\left(q^{d}-1\right) z$ for some $z \in Y$, so that $y_{2}$ and $y_{2}^{\prime}$ are representatives for $\bar{y}_{2}$. Since $Y^{\#} \subset n Y$ and $n$ divides $\left(q^{d}-1\right)$, we find that $y_{2} \in Y^{\#}$ if and only if $y_{2}^{\prime} \in Y^{\#}$.

Similarly, we find that $\delta_{q} y_{2}^{\prime}=\delta_{q} y_{2}+\left(q^{d}-1\right) \delta_{q} z$. By Lemma 5.6, $\delta_{q} z \in Y^{\Gamma \#}$. It follows that $\delta_{q} y_{2} \in\left(q^{d}-1\right) Y^{\Gamma \#}$ if and only if $\delta_{q} y_{2}^{\prime} \in\left(q^{d}-1\right) Y^{\Gamma \#}$.

The above corollary implies that $y_{1}(\varpi) \in Z^{\dagger}\left(T_{F}\right)$ for a given $y_{1} \in Y$ if and only if $y_{1} \in Y^{\# \Gamma}$. It also implies the following:

Corollary 5.10. Suppose that $\bar{y}_{2} \in \bar{Y}$. Then $\bar{y}_{2}\left(\vartheta_{L}\right) \in Z^{\dagger}\left(T_{F}\right)$ if and only if

$$
\bar{y}_{2} \in \operatorname{Im}\left(\operatorname{Tr}_{q}\left(\overline{Y^{\Gamma \#}}\right) \rightarrow \bar{Y}\right) .
$$

Proof. The previous corollary implies that $\bar{y}_{2}\left(\vartheta_{L}\right) \in Z^{\dagger}\left(T_{F}\right)$ if and only if

(1) $y_{2} \in Y^{\#}$ for some (equivalently, every) representative $y_{2}$ of $\bar{y}_{2}$, and

(2) $\delta_{q} y_{2} \in\left(q^{d}-1\right) Y^{\Gamma \#}$ for some (equivalently, every) representative $y_{2}$ of $\bar{y}_{2}$.

Given these conditions and a representative $y_{2}$ of $\bar{y}_{2}$, there exists $w \in Y^{\Gamma \#}$ such that $\delta_{q}\left(y_{2}\right)=\left(q^{d}-1\right) w$. Hence $\delta_{q}\left(y_{2}\right)=\delta_{q} \operatorname{Tr}_{q}(w)$. The injectivity of $\delta_{q}$ implies that $y_{2}=\operatorname{Tr}_{q}(w)$. It follows that $\bar{y}_{2}$ is the image of $\operatorname{Tr}_{q}(\bar{w})$ in $\bar{Y}$. Hence, conditions (1) and (2) imply the one condition (5-2) of this corollary.

Conversely, suppose that Equation (5-2) is satisfied. Then we may choose $w \in Y^{\Gamma \#}$ such that $\bar{y}_{2}$ equals the image of $\operatorname{Tr}_{q}(\bar{w})$ in $\bar{Y}$. Thus $y_{2}=\operatorname{Tr}_{q}(w)$ is a representative for $\bar{y}_{2}$ in $\bar{Y}$. Since $\operatorname{Tr}_{q}\left(Y^{\Gamma \#}\right) \subset Y^{\#}$ by Lemma 5.6, condition (1) is satisfied. Since $\delta_{q} y_{2}=\operatorname{Tr}_{q} \delta_{q} w=\left(q^{d}-1\right) w$, condition (2) is satisfied as well. Therefore, $\bar{y}_{2}\left(\vartheta_{L}\right) \in Z^{\dagger}\left(T_{F}\right)$. 
The image of an isogeny. For split metaplectic tori, we found a useful characterization of $Z^{\dagger}\left(T_{F}\right)$ as the image of an isogeny on $F$-rational points. The same isogeny makes sense for nonsplit tori; however there is a small but important difference between the image of the isogeny and $Z^{\dagger}\left(T_{F}\right)$. We view this difference as accounting for "packets" of representations of metaplectic tori, with the same parameter.

Consider the inclusion of $\mathbb{Z}[\Gamma]$-modules $\imath: Y^{\#} \hookrightarrow Y$. Note that we use the fact that $Q$ is a $\Gamma$-invariant quadratic form, so that $Y^{\#}$ is a $\mathbb{Z}[\Gamma]$-submodule. This inclusion corresponds to an isogeny $\iota: \boldsymbol{T}^{\#} \rightarrow \boldsymbol{T}$ of algebraic tori over $F$. Our description of the $F$-rational and $L$-rational points for $\boldsymbol{T}$ is also valid, mutatis mutandis, for $\boldsymbol{T}^{\#}$. When $y \in Y^{\#}$ and $u \in L^{\times}$, we simply write $(y \otimes u)$ for the corresponding element of $\boldsymbol{T}^{\#}(L) \equiv Y^{\#} \otimes L^{\times}$. We choose this notation rather than $y(u)$ since we do not wish to confuse cocharacters of $\boldsymbol{T}$ with cocharacters of $\boldsymbol{T}^{\#}$. Since $Y^{\#}$ is a $\mathbb{Z}[\Gamma]$-module, we find this:

Proposition 5.11. The torus $\boldsymbol{T}^{\#}$ splits over an unramified extension of $F$. Suppose that $y_{1}, y_{2} \in Y^{\#}$. Then $\left(y_{1} \otimes \varpi\right)\left(y_{2} \otimes \vartheta_{L}\right) \in T^{\#}=\boldsymbol{T}^{\#}(F)$ if and only if

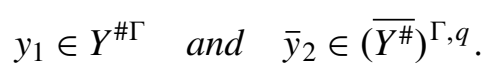

The isogeny $\iota$ acts on $L$-rational points by

$$
\boldsymbol{\iota}(y \otimes u)=y(u) \quad \text { for all } y \in Y^{\#}, u \in L^{\times} \text {and }(y \otimes u) \in \boldsymbol{T}^{\#}(L) .
$$

Proposition 5.12. Suppose that $y_{1} \in Y, \bar{y}_{2} \in \bar{Y}$, and $t^{1} \in T_{L}^{1}$. Then $y_{1}(\varpi) \bar{y}_{2}(\vartheta) t^{1}$ is an element of the image of $l: \boldsymbol{T}^{\#}(F) \rightarrow \boldsymbol{T}(F)$ if and only if

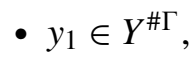

- there exists a $y_{2} \in Y^{\#}$ representing $\bar{y}_{2}$ such that $\delta_{q} y_{2} \in\left(q^{d}-1\right) Y^{\#}$, and

- $t^{1} \in T_{F}^{1}$.

Proof. Since $(n, p)=1$, the image of $\imath$ contains $T_{F}^{1}$. It suffices only to consider the images $\imath\left(\left(y_{1} \otimes \varpi\right)\left(y_{2} \otimes \vartheta_{L}\right)\right)$ for all $y_{1} \in Y^{\# \Gamma}$ and $y_{2} \in Y^{\#}$ such that $\bar{y}_{2} \in\left(\overline{Y^{\#}}\right)^{\Gamma, q}$.

Corollary 5.13. Suppose that $\bar{y}_{2} \in \bar{Y}$. Then $\bar{y}_{2}\left(\vartheta_{L}\right) \in l\left(\boldsymbol{T}^{\#}(F)\right)$ if and only if

$$
\bar{y}_{2} \in \operatorname{Im}\left(\operatorname{Tr}_{q}\left(\overline{Y^{\#}}\right) \rightarrow \bar{Y}\right) \text {. }
$$

Proof. If $\bar{y}_{2} \in \operatorname{Im}\left(\operatorname{Tr}_{q}\left(\overline{Y^{\#}}\right) \rightarrow \bar{Y}\right)$, there exists an element $y_{3} \in Y^{\#}$ such that $\bar{y}_{2}$ equals the image of $\operatorname{Tr}_{q}\left(y_{3}\right)$ in $\bar{Y}$. If $y_{2}=\operatorname{Tr}_{q}\left(y_{3}\right)$, then $y_{2}$ is a representative for $\bar{y}_{2}$ in $Y$. Note that $y_{2} \in Y^{\#}$ since $y_{3} \in Y^{\#}$. Also $\delta_{q} y_{2}=\delta_{q} \operatorname{Tr}_{q}\left(y_{3}\right)=\left(q^{d}-1\right) y_{3} \in\left(q^{d}-1\right) Y^{\#}$. Hence $\bar{y}_{2}\left(\vartheta_{L}\right) \in \imath\left(\boldsymbol{T}^{\#}(F)\right)$ by the previous proposition.

Conversely, suppose that $\bar{y}_{2}\left(\vartheta_{L}\right) \in \imath\left(\boldsymbol{T}^{\#}(F)\right)$. By the previous proposition, there exists a representative $y_{2}$ of $\bar{y}_{2}$ in $Y$ such that $y_{2} \in Y^{\#}$ and $\delta_{q}\left(y_{2}\right) \in\left(q^{d}-1\right) Y^{\#}$. It 
follows that $\delta_{q}\left(y_{2}\right)=\delta_{q} \operatorname{Tr}_{q}\left(y_{3}\right)$ for some $y_{3} \in Y^{\#}$. Hence $y_{2}=\operatorname{Tr}_{q}\left(y_{3}\right)$. Hence $\bar{y}_{2}$ is contained in the image of $\operatorname{Tr}_{q}\left(\overline{Y^{\#}}\right)$ in $\bar{Y}$.

The packet group. From the previous two sections, we have described the groups $Z^{\dagger}\left(T_{F}\right)$ and $\iota\left(\boldsymbol{T}^{\#}(F)\right)$. They are quite similar, with one exception. For given $\bar{y}_{2} \in \bar{Y}$, we have

- $\bar{y}_{2}\left(\vartheta_{L}\right) \in Z^{\dagger}\left(T_{F}\right)$ if and only if $\bar{y}_{2} \in \operatorname{Im}\left(\operatorname{Tr}_{q}\left(\overline{Y^{\Gamma \#}}\right) \rightarrow \bar{Y}\right)$, and

- $\bar{y}_{2}\left(\vartheta_{L}\right) \in \imath\left(\boldsymbol{T}^{\#}(F)\right)$ if and only if $\bar{y}_{2} \in \operatorname{Im}\left(\operatorname{Tr}_{q}\left(\overline{Y^{\#}}\right) \rightarrow \bar{Y}\right)$.

Define a finite group $P_{\theta_{1}}^{\dagger}$ by

$$
P_{\theta_{1}}^{\dagger}=\operatorname{Im}\left(\operatorname{Tr}_{q}\left(\overline{Y^{\Gamma \#}}\right) \rightarrow \bar{Y}\right) / \operatorname{Im}\left(\operatorname{Tr}_{q}\left(\overline{Y^{\#}}\right) \rightarrow \bar{Y}\right) .
$$

It follows from Proposition 5.12 and Corollary 5.9 that there is a natural short exact sequence

$$
1 \rightarrow \imath\left(\boldsymbol{T}^{\#}(F)\right) \rightarrow Z^{\dagger}\left(T_{F}\right) \rightarrow P_{\theta_{\mathbf{l}}}^{\dagger} \rightarrow 1 .
$$

However, this sequence depends upon the choice of generator $\theta_{\mathbf{l}}$ of $\mathbf{l}^{\times}$. We identify $P_{\theta_{1}}^{\dagger}$ here so that this sequence is independent of the choice of generator.

The $\mathbb{Z}[\Gamma]$-modules $Y^{\Gamma \#}$ and $Y^{\#}$ correspond to a pair $\overline{\boldsymbol{T}}^{\Gamma \#}$ and $\overline{\boldsymbol{T}}^{\#}$ of $\mathbf{f}$-tori that split over l. Moreover, the inclusions $Y^{\#} \subset Y^{\Gamma \#} \subset Y$ correspond to $\mathbf{f}$-isogenies of f-tori via $\overline{\boldsymbol{T}}^{\#} \rightarrow \overline{\boldsymbol{T}}^{\Gamma \#} \rightarrow \overline{\boldsymbol{T}}$. The choice of generator $\theta_{\mathbf{l}}$ of $\mathbf{I}^{\times}$corresponds to the identifications

$$
\overline{\boldsymbol{T}}^{\Gamma \#}(\mathbf{l}) \equiv \overline{Y^{\Gamma \#}} \quad \text { and } \quad \overline{\boldsymbol{T}}^{\#}(\mathbf{l}) \equiv \overline{Y^{\#}} .
$$

Furthermore, the trace map $\operatorname{Tr}_{q}$ corresponds to the norm maps. For example, there is a commutative diagram

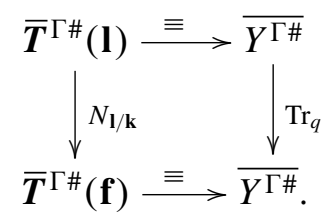

Now, Lang's theorem [1956] implies that the norm map is surjective. In other words, the commutative diagram above yields the identifications

$$
\overline{\boldsymbol{T}}^{\Gamma \#}(\mathbf{f}) \equiv \operatorname{Tr}_{q} \overline{Y^{\Gamma \#}} \quad \text { and } \quad \overline{\boldsymbol{T}}^{\#}(\mathbf{f}) \equiv \operatorname{Tr}_{q} \overline{Y^{\#}} .
$$

A proposition follows:

Proposition 5.14. Define a finite group $P^{\dagger}$ by

$$
P^{\dagger}=\operatorname{Im}\left(\overline{\boldsymbol{T}}^{\Gamma \#}(\mathbf{f}) \rightarrow \overline{\boldsymbol{T}}(\mathbf{f})\right) / \operatorname{Im}\left(\overline{\boldsymbol{T}}^{\#}(\mathbf{f}) \rightarrow \overline{\boldsymbol{T}}(\mathbf{f})\right) .
$$

Then $P_{\theta_{1}}^{\dagger} \cong P^{\dagger}$. 
The construction of the group $P^{\dagger}$ does not depend upon the choice of generator $\theta_{\mathbf{l}}$. This yields a canonical short exact sequence

$$
1 \rightarrow \imath\left(\boldsymbol{T}^{\#}(F)\right) \rightarrow Z^{\dagger}\left(T_{F}\right) \rightarrow P^{\dagger} \rightarrow 1,
$$

which depends neither on the choice of uniformizing element $\varpi$ nor on the choice of generator $\theta_{\mathbf{l}}$.

The main theorem of Langlands [1997], which parameterizes smooth characters of tori over local fields, determines isomorphisms

$$
\mathscr{X}\left(T_{F}\right) \cong H_{c}^{1}\left(W_{L / F}, \hat{\mathcal{T}}\right) \text { and } \mathscr{X}\left(T_{F}^{\#}\right) \cong H_{c}^{1}\left(\mathscr{W}_{L / F}, \hat{\mathscr{T}}^{\#}\right) .
$$

As before, the characters of the image of an isogeny can be parameterized cohomologically:

Proposition 5.15. The Langlands parameterization yields a finite-to-one parameterization of the smooth characters of $Z^{\dagger}\left(T_{F}\right)$ :

$$
1 \rightarrow \mathscr{X}\left(P^{\dagger}\right) \rightarrow \mathscr{X}\left(Z^{\dagger}\left(T_{F}\right)\right) \rightarrow H_{c}^{1}\left(\mathscr{W}_{L / F}, \hat{\mathfrak{T}}\right) / H_{c}^{1}\left(\mathscr{W}_{L / F}, \hat{\mathfrak{T}} \rightarrow \hat{\mathfrak{T}}^{\#}\right) \rightarrow 1 .
$$

Remark 5.16. To view $H_{c}^{1}\left(\mathcal{W}_{L / F}, \hat{\mathfrak{T}} \rightarrow \hat{\mathscr{T}}^{\#}\right)$ as a subgroup of $H_{c}^{1}\left(\mathcal{W}_{L / F}, \hat{\mathfrak{T}}\right)$ as above, we must know that the map $H_{c}^{0}\left(\mathcal{W}_{L / F}, \hat{\mathfrak{T}}\right) \rightarrow H_{c}^{0}\left(\mathcal{W}_{L / F}, \hat{\mathfrak{T}}^{\#}\right)$ is surjective. This follows from the identifications

$$
H_{c}^{0}\left(W_{L / F}, \hat{\mathscr{T}}\right) \equiv \operatorname{Hom}_{\mathbb{Z}}\left(Y^{\Gamma}, \mathbb{C}^{\times}\right) \quad \text { and } \quad H_{c}^{0}\left(\mathcal{W}_{L / F}, \hat{\mathscr{T}}^{\#}\right) \equiv \operatorname{Hom}_{\mathbb{Z}}\left(Y^{\# \Gamma}, \mathbb{C}^{\times}\right)
$$

and the fact that $Y^{\# \Gamma}$ has finite index in $Y^{\Gamma}$.

This leads directly, via a Stone-von Neumann theorem, to a main theorem for tame covers of unramified tori:

Theorem 5.17. Suppose that we have tame metaplectic cover of an unramified torus given by

$$
1 \rightarrow \mu_{n} \rightarrow \tilde{T} \rightarrow T \rightarrow 1
$$

Then, with the sublattices $Y^{\#} \subset Y^{\Gamma \#} \subset Y$ defined as before and the resulting isogenies $\boldsymbol{T}^{\#} \rightarrow \boldsymbol{T}^{\Gamma \#} \rightarrow \boldsymbol{T}$ of unramified tori, we find that

- there is a finite-to-one surjective map

$$
\Phi: \Phi_{\epsilon}(\tilde{T}) \rightarrow H_{c}^{1}\left(W_{L / F}, \hat{\mathscr{T}}\right) / H_{c}^{1}\left(W_{L / F}, \hat{\mathscr{T}} \rightarrow \hat{\mathscr{T}}^{\#}\right)
$$

intertwining the natural action of $H^{1}\left(W_{L / F}, \hat{\mathscr{T}}\right)$, and

- the fibres of this map are torsors for the finite group $\mathscr{X}\left(P^{\dagger}\right)$, where

$$
P^{\dagger}=\operatorname{Im}\left(\overline{\boldsymbol{T}}^{\Gamma \#}(\mathbf{f}) \rightarrow \overline{\boldsymbol{T}}(\mathbf{f})\right) / \operatorname{Im}\left(\overline{\boldsymbol{T}}^{\#}(\mathbf{f}) \rightarrow \overline{\boldsymbol{T}}(\mathbf{f})\right) .
$$


Remark 5.18. We do not know if a parameterization such as that above holds for general metaplectic tori over local fields. Namely, we have not been able to describe the center of such metaplectic tori for the case in which $\boldsymbol{T}$ is ramified or an unramified torus but the cover is not tame. We hope that such a parameterization is possible, though the packets might be substantially different.

Remark 5.19. In proving the previous theorem, we chose a uniformizing element $\varpi \in F^{\times}$and a root of unity $\theta_{L}$. However, this choice does not have any effect on the parameterization given above. The sublattices $Y^{\#}$ and $Y^{\Gamma \#}$ clearly do not depend upon such a choice. Moreover, the action of $\mathscr{X}\left(P^{\dagger}\right)$ on the fibres of $\Phi$ does not depend on such a choice.

\section{Pseudospherical and pseudotrivial representations}

We maintain all of the conventions of the previous section. In particular, we have a tame metaplectic cover of an unramified torus given by

$$
1 \rightarrow \mu_{n} \rightarrow \tilde{T}_{F} \rightarrow T_{F} \rightarrow 1 \text {. }
$$

We have shown that the irreducible genuine representations of $\tilde{T}_{F}$ can be parameterized by the points of a homogeneous space on which $H^{1}\left(\mathcal{W}_{L / F}, \hat{\mathfrak{T}}\right)$ acts transitively. However, such a parameterization is not unique; one must choose a base point in the space of irreducible genuine representations of $\tilde{T}_{F}$ in order to choose a specific morphism

$$
\Phi: \mathscr{I}_{\epsilon}\left(\tilde{T}_{F}\right) \rightarrow H^{1}\left(\mathscr{W}_{L / F}, \hat{\mathscr{T}}\right) / H^{1}\left(\mathcal{W}_{L / F}, \hat{\mathscr{T}} \rightarrow \hat{\mathscr{T}}^{\#}\right)
$$

of homogeneous spaces.

In this section, we discuss the data that determines such base points. Such choices arise frequently in treatments of metaplectic groups, often as choices of square roots of -1 in $\mathbb{C}$.

The residual extension. Recall that the unramified torus $\boldsymbol{T}$ has a smooth model $\underline{T}$ over $\mathscr{O}_{F}$, and $T_{F}^{\circ}=\underline{\boldsymbol{T}}\left(\mathrm{O}_{F}\right)$. In this case, $T_{F}^{\circ}$ is the maximal compact subgroup of $T$, and we let $\tilde{T}_{F}^{\circ}$ be its preimage in $\tilde{T}_{F}$. Also, $\overline{\boldsymbol{T}}$ denotes the special fibre of $\underline{\boldsymbol{T}}$ that is a torus over $\mathbf{f}$. Recall that $\boldsymbol{T}^{\prime}$ is a central extension of $\boldsymbol{T}$ by $\boldsymbol{K}_{2}$. Pushing forward via the tame symbol led to the tame central extension

$$
1 \rightarrow \mathbf{f}^{\times} \rightarrow T_{F}^{t} \rightarrow T_{F} \rightarrow 1 .
$$

We write $T_{F}^{t \circ}$ for the preimage of $T_{F}^{\circ}$ in $T_{F}^{t}$.

Deligne and Brylinski, in [2001, Section 12.11], construct an extension $\overline{\boldsymbol{T}}^{\prime}$ of $\overline{\boldsymbol{T}}$ by $\boldsymbol{G}_{m}$ (in the category of groups over $\mathbf{f}$ ). We call $\overline{\boldsymbol{T}}^{\prime}$ the residual extension 
associated to $\boldsymbol{T}^{\prime}$. The residual extension fits into the commutative diagram

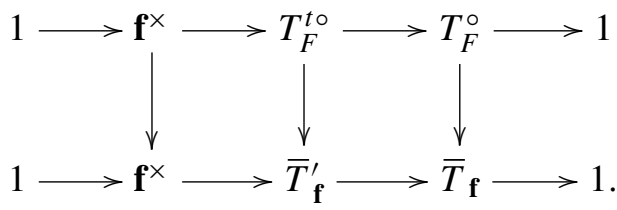

Here, the map from $\mathbf{f}^{\times}$to itself is the identity, the map from $T_{F}^{\circ}$ to $\bar{T}_{\mathbf{f}}$ is the reduction map, and the diagram identifies the top row with the pullback of the bottom row via reduction.

As an extension of $\overline{\boldsymbol{T}}$ by $\boldsymbol{G}_{m}$ over $\mathbf{f}$, the group $\overline{\boldsymbol{T}}^{\prime}$ is an algebraic torus over $\mathbf{f}$. Note that the category of extensions of $\overline{\boldsymbol{T}}$ by $\boldsymbol{G}_{m}$, in the category of groups over $\mathbf{f}$, is equivalent to the category of extensions of $Y$ by $\mathbb{Z}$, in the category of $\mathbb{Z}[\Gamma]$-modules (where $\mathbb{Z}$ is given the trivial module structure). In this way, the construction of [Brylinski and Deligne 2001, Section 12.11] associates an extension of $Y$ by $\mathbb{Z}$ to any extension of an unramified torus $\boldsymbol{T}$ by $\boldsymbol{K}_{2}$.

Remark 6.1. Recall that $\tilde{Y}$ is a $\Gamma$-equivariant extension of $Y$ by $L^{\times}$, constructed as a functorial invariant of the extension $\boldsymbol{T}^{\prime}$ of $\boldsymbol{T}$ by $\boldsymbol{K}_{2}$. Let $Y^{\prime}$ be the extension of $Y$ by $\mathbb{Z}$, obtained by pushing forward $\tilde{Y}$ via the valuation map $L^{\times} \rightarrow \mathbb{Z}$, that is,

$$
0 \rightarrow \mathbb{Z} \rightarrow Y^{\prime} \rightarrow Y \rightarrow 0 .
$$

We do not know whether this extension is naturally isomorphic to the exact sequence of cocharacter groups of the residual extension of tori described above

Definition 6.2. Let $\operatorname{Spl}\left(\overline{\boldsymbol{T}}^{\prime}\right)$ denote the set of splittings, in the category of algebraic groups over $\mathbf{f}$, of the short exact sequence

$$
1 \rightarrow \boldsymbol{G}_{m} \rightarrow \overline{\boldsymbol{T}}^{\prime} \rightarrow \overline{\boldsymbol{T}} \rightarrow 1 .
$$

We say the extension $\boldsymbol{T}^{\prime}$ of the unramified torus $\boldsymbol{T}$ is a residually split extension if $\operatorname{Spl}\left(\overline{\boldsymbol{T}}^{\prime}\right)$ is nonempty.

In particular, if $\boldsymbol{T}$ is a split torus, then $\boldsymbol{T}^{\prime}$ is residually split.

Proposition 6.3. If $\operatorname{Spl}\left(\overline{\boldsymbol{T}}^{\prime}\right)$ is nonempty, then $\operatorname{Spl}\left(\overline{\boldsymbol{T}}^{\prime}\right)$ is a torsor for the abelian group $X^{\Gamma}$.

Proof. Any two algebraic splittings are related by an element of $\operatorname{Hom}_{\mathbf{f}}\left(\overline{\boldsymbol{T}}, \boldsymbol{G}_{m}\right)$. This group may be identified with the $\Gamma$-fixed characters of $\boldsymbol{T}$.

Pseudospherical representations. Suppose now that $\boldsymbol{T}^{\prime}$ is a residually split extension of $\boldsymbol{T}$ by $\boldsymbol{K}_{2}$. Fix a splitting $s \in \operatorname{Spl}\left(\overline{\boldsymbol{T}}^{\prime}\right)$. The splitting lifts to a splitting $\sigma: T_{F}^{\circ} \rightarrow T_{F}^{t \circ}$. Pushing forward via the $m$-th power map, we may also view $\sigma$ as a splitting $T_{F}^{\circ} \rightarrow \tilde{T}_{F}^{\circ}$. From such a splitting $s$, we let $\theta_{s}^{\circ}: \tilde{T}_{F}^{\circ} \rightarrow \mathbb{C}^{\times}$denote the 
character obtained by projecting onto $\mu_{n}$ (via the splitting $\sigma$ ) and then applying the injective homomorphism $\epsilon: \mu_{n} \rightarrow \mathbb{C}^{\times}$.

Let $Z_{\tilde{T}_{F}}\left(\tilde{T}_{F}^{\circ}\right)$ be the centralizer of $\tilde{T}_{F}^{\circ}$ in $\tilde{T}_{F}$.

Proposition 6.4. The group $Z_{\tilde{T}_{F}}\left(\tilde{T}_{F}^{\circ}\right)$ is the preimage of a subgroup $Z_{T_{F}}^{\dagger}\left(T_{F}^{\circ}\right) \subset T_{F}$. Consider the valuation map

$$
\text { val: } T_{F} \rightarrow Y^{\Gamma}
$$

whose kernel is $T_{F}^{\circ}$. Then $Z_{T_{F}}^{\dagger}\left(T_{F}^{\circ}\right)$ is equal to the preimage of $Y^{\# \Gamma}$.

Proof. Since $Z_{T_{F}}^{\dagger}\left(T_{F}^{\circ}\right) \supset T_{F}^{\circ}$, it suffices to identify the set of $y \in Y^{\Gamma}$ such that

$$
C_{L}\left(y(\varpi), \bar{y}^{\prime}\left(\vartheta_{L}\right)\right)=1 \quad \text { for all } \bar{y}^{\prime} \in \bar{Y}^{\Gamma, q} .
$$

In fact, the set of such $y$ has been identified in the proofs of Theorems 5.7 and 5.8. The above condition is satisfied if and only if $y \in Y^{\# \Gamma}$.

Corollary 6.5. The group $Z_{\tilde{T}_{F}}\left(\tilde{T}_{F}^{\circ}\right)$ is abelian.

Proof. As $Z_{\tilde{T}_{F}}\left(\tilde{T}_{F}^{\circ}\right)$ is the centralizer of the abelian group $\tilde{T}_{F}^{\circ}$, it suffices to prove that $C\left(y(\varpi), y^{\prime}(\varpi)\right)=1$ for all $y, y^{\prime} \in Y^{\# \Gamma}$. This is proved in the beginning of the proof of Theorem 5.8.

Directly following [Savin 2004, Section 4], we find this:

Proposition 6.6. There is a natural bijection between the following two sets:

- The set $\Phi_{s, \epsilon}^{\mathrm{sph}}\left(\tilde{T}_{F}\right)$ of pseudospherical irreducible representations of $\tilde{T}_{F}$ (for the splitting $s$ ). These are the genuine irreducible representations of $\tilde{T}_{F}$ whose restriction to $\tilde{T}_{F}^{\circ}$ via the splitting s contains a nontrivial $\theta_{s}^{\circ}$-isotypic component.

- The set of extensions of $\theta_{s}^{\circ}$ to the group $Z_{\tilde{T}_{F}}\left(\tilde{T}_{F}^{\circ}\right)$.

Namely, if $(\pi, V)$ is a pseudospherical irreducible representation, its $\theta_{s}^{\circ}$-isotypic subrepresentation is an extension of $\theta_{s}^{\circ}$ to the group $Z_{\tilde{T}_{F}}\left(\tilde{T}_{F}^{\circ}\right)$. Conversely, given such an extension $\theta_{s}^{1}$ of $\theta_{s}^{\circ}$ to a character of $Z_{\tilde{T}_{F}}\left(\tilde{T}_{F}^{\circ}\right)$, the induced representation $\operatorname{Ind}_{Z_{\tilde{T}_{F}}\left(\tilde{T}_{F}^{\circ}\right)} \theta_{s}^{1}$ is a pseudospherical irreducible representation.

One may rephrase the bijection above slightly: the splitting $s$ yields an injective homomorphism from $T_{F}^{\circ}$ onto a normal subgroup of $Z_{\tilde{T}_{F}}\left(\tilde{T}_{F}^{\circ}\right)$. This fits into a 
commutative diagram

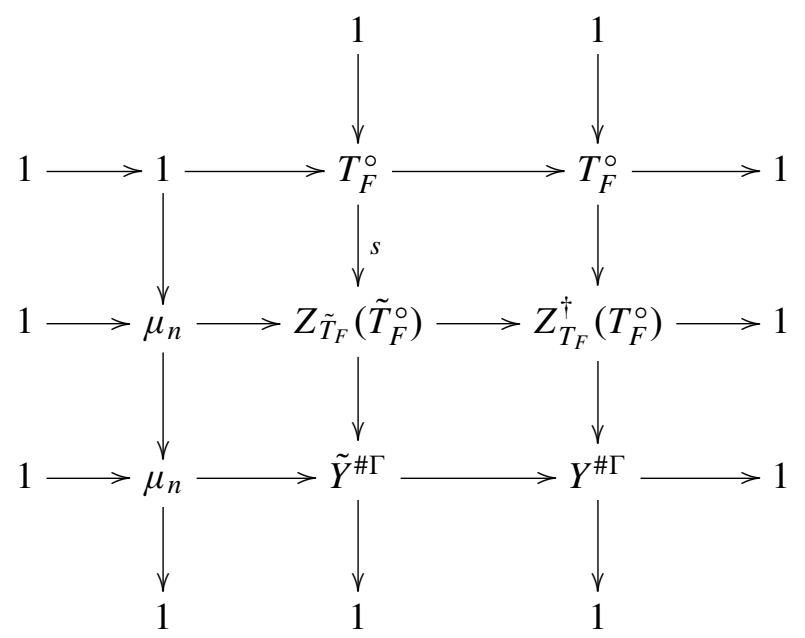

with exact rows and columns. Hence, the splitting $s$ determines an extension $\tilde{Y}^{\# \Gamma}$ of $Y^{\# \Gamma}$ by $\mu_{n}$. A standard diagram chase now yields this:

Proposition 6.7. There is a natural bijection

$$
g_{s, \epsilon}^{\mathrm{sph}}\left(\tilde{T}_{F}\right) \leftrightarrow \mathscr{X}_{\epsilon}\left(\tilde{Y}^{\# \Gamma}\right) .
$$

Corollary 6.8. The space $\mathscr{I}_{s, \epsilon}^{\mathrm{sph}}\left(\tilde{T}_{F}\right)$ is naturally a torsor for the complex algebraic torus $\mathscr{L}\left(Y^{\# \Gamma}\right)$.

Remark 6.9. One may view $\mathscr{X}_{\epsilon}\left(\tilde{Y}^{\# \Gamma}\right)$ as the set of irreducible representations of a "quantum torus". Indeed, the ring

$$
\mathbb{C}_{\epsilon}\left[\tilde{Y}^{\# \Gamma}\right]=\mathbb{C}\left[\tilde{Y}^{\# \Gamma}\right] /\langle\zeta-\epsilon(\zeta)\rangle_{\zeta \in \mu_{n}},
$$

can be viewed as (the coordinate ring of) a quantum complex torus. This torus, which we call $\hat{\mathscr{T}}_{\epsilon}^{\# \Gamma}$, is the quantization of a complex torus, at a root of unity. Quasicoherent sheaves on this quantum torus (that is, modules over its coordinate ring) correspond naturally to pseudospherical representations of $\tilde{T}_{F}$.

Pseudotrivial representations. In many practical situations, the extension $\tilde{Y}^{\# \Gamma}$ of $Y^{\# \Gamma}$ by $\mu_{n}$ splits over a quite large submodule of $Y^{\# \Gamma}$. For example, in many cases, the extension splits over $Y^{\# \Gamma} \cap 2 Y$.

Suppose that $V \subset Y^{\# \Gamma}$ is a finite index subgroup, endowed with a splitting $v$ of the resulting exact sequence

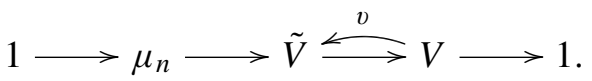


Let $U=Y^{\# \Gamma / V}$ denote the quotient. The splitting $v$ yields an extension of finite abelian groups given by $1 \rightarrow \mu_{n} \rightarrow \tilde{U} \rightarrow U \rightarrow 1$.

Pulling back yields natural inclusions $\mathscr{X}_{\epsilon}(\tilde{U}) \hookrightarrow \mathscr{X}_{\epsilon}\left(\tilde{Y}^{\# \Gamma}\right) \equiv \mathscr{I}_{s, \epsilon}^{\mathrm{sph}}\left(\tilde{T}_{F}\right)$.

Therefore, within the set of pseudospherical representations of $\tilde{T}_{F}$, we find a finite set of "pseudotrivial" representations (relative to the choice of splitting subgroup $(V, v)$ of $Y^{\# \Gamma \text { : }}$

Definition 6.10. The genuine pseudotrivial representations of $\tilde{T}_{F}$ are those irreducible pseudospherical genuine representations that are in the image of $\mathscr{X}_{\epsilon}(\tilde{U})$. This definition depends upon the choice of

- the splitting $s$ (to determine the pseudospherical representations), and

- the splitting subgroup $(V, v)$ (to determine the pseudotrivial representations).

Remark 6.11. Most often, one chooses a pseudotrivial "base point" in the space $\Phi_{\epsilon}\left(\tilde{T}_{F}\right)$. Very often (see the examples of [Savin 2004]), $\tilde{U}$ is a finite abelian group of exponent 4. It follows that pseudotrivial representations may often be given by specifying certain characters of an abelian group of exponent 4 . This explains why choosing fourth roots of unity is often necessary in work on metaplectic groups.

\section{Tori over $\mathbb{R}$}

In this section, we fix these notations:

- $\boldsymbol{T}$ will be a torus over $\mathbb{R}$, and $\Gamma=\operatorname{Gal}(\mathbb{C} / \mathbb{R})=\{1, \gamma\}$. $X$ and $Y$ will be the resulting character and cocharacter groups, viewed as $\mathbb{Z}[\Gamma]$-modules.

- $\boldsymbol{T}^{\prime}$ will be an extension of $\boldsymbol{T}$ by $\boldsymbol{K}_{2}$ in $\mathfrak{G}_{\mathfrak{R}}$.

- $(Q, \tilde{Y})$ will be the Deligne-Brylinski invariants of $\boldsymbol{T}^{\prime}$, and $B$ will be the symmetric bilinear form associated to $Q$.

- We fix $n=2$, so that $\mathbb{R}$ has enough $n$-th roots of unity.

- If $W$ is a subgroup of $Y$, then we write $W^{\Gamma}$ for the subgroup of $\Gamma$-fixed elements of $W$. We also define

$$
W^{\#}=\{y \in Y \text { such that } B(y, w) \in 2 \mathbb{Z} \text { for all } w \in W\} .
$$

- $\epsilon: \mu_{2}(\mathbb{R}) \rightarrow \mathbb{C}^{\times}$will be the unique injective character.

- We view $T=\boldsymbol{T}(\mathbb{R})$ as a real Lie group. We write $T^{\circ}$ for the connected component of the identity element, and $\pi_{0} T$ for the component group of $T$.

- The extension $\boldsymbol{T}^{\prime}$, and the quadratic Hilbert symbol, yields an extension of Lie groups given by

$$
1 \rightarrow \mu_{2} \rightarrow \tilde{T} \rightarrow T \rightarrow 1
$$


We are interested in parameterizing the irreducible genuine representations of $\tilde{T}$, which, as before, we call $\Phi_{\epsilon}(\tilde{T})$.

Structure of metaplectic tori over $\mathbb{R}$. There is a short exact sequence

$$
1 \rightarrow T^{\circ} \rightarrow T \rightarrow \pi_{0} T \rightarrow 1
$$

of Lie groups. Let $\boldsymbol{T}^{\Gamma}$ be the split real torus with cocharacter group $Y^{\Gamma}$. Let $T^{\Gamma}$ denote the real points of $\boldsymbol{T}^{\Gamma}$. Then, we find that $\pi_{0} T^{\Gamma}$ is canonically isomorphic to $\overline{Y^{\Gamma}}=Y^{\Gamma} \otimes_{\mathbb{Z}} \mu_{2} \equiv Y^{\Gamma} / 2 Y^{\Gamma}$. Moreover, the inclusion of $\mathbb{R}$-tori from $\boldsymbol{T}^{\Gamma}$ into $\boldsymbol{T}$ induces a surjection $\pi_{0} T^{\Gamma} \rightarrow \pi_{0} T$ of component groups. Therefore, every element $t$ of $T$ has a (often nonunique) decomposition $t=t^{\circ} \bar{y}(-1)$ for some $t^{\circ} \in T^{\circ}$ and $\bar{y} \in \overline{Y^{\Gamma}}$. In other words, there is a natural surjective homomorphism $\overline{Y^{\Gamma}} \rightarrow \pi_{0} T$.

Now, we consider the metaplectic cover $1 \rightarrow \mu_{2} \rightarrow \tilde{T} \rightarrow T \rightarrow 1$ of $T$. The commutator $C: T \times T \rightarrow \mu_{2}$ is bimultiplicative and continuous. It follows that the commutator is trivial when either of its inputs is in $T^{\circ}$. Hence:

Proposition 7.1. $T^{\circ}$ is a subgroup of $Z^{\dagger}(T)$.

Description of the center. It follows from the previous proposition that to describe $Z^{\dagger}(T)$, it suffices to describe its image in $T / T^{\circ}$. Hence, it suffices to determine the $\bar{y} \in \overline{Y^{\Gamma}}$ for which $\bar{y}(-1) \in Z^{\dagger}(T)$. We must be able to compute the commutator $C\left(\bar{y}(-1), \bar{y}^{\prime}(-1)\right)$ for arbitrary $\bar{y}, \bar{y}^{\prime} \in \overline{Y^{\Gamma}}$.

Here, we note that such elements $\bar{y}(-1)$ and $\bar{y}^{\prime}(-1)$ are contained in the real points of the maximal $\mathbb{R}$-split subtorus $\boldsymbol{T}^{\Gamma} \hookrightarrow \boldsymbol{T}$. Restricting the central extension of $\boldsymbol{T}$ by $\boldsymbol{K}_{2}$ to the split subtorus $\boldsymbol{T}^{\Gamma}$, the formula of [Brylinski and Deligne 2001, Corollary 3.14$]$ is valid for computing commutators.

Proposition 7.2. If $y, y^{\prime} \in Y^{\Gamma}$, then $C\left(\bar{y}(-1), \bar{y}^{\prime}(-1)\right)=(-1)^{B\left(y, y^{\prime}\right)}$.

Proof. This follows directly from [Brylinski and Deligne 2001, Corollary 3.14] and the Hilbert symbol over $\mathbb{R}$, which satisfies $(-1,-1)_{\mathbb{R}, 2}=-1$.

Proposition 7.3. Given $\bar{y} \in \overline{Y^{\Gamma}}$, we have $\bar{y}(-1) \in Z^{\dagger}(T)$ if and only if every representative $y$ of $\bar{y}$ in $Y$ satisfies $y \in Y^{\Gamma \# \Gamma}$.

Proof. Suppose $\bar{y}, \bar{y}^{\prime} \in \overline{Y^{\Gamma}}$. Let $y$ be a representative of $\bar{y}$ in $Y$. The commutator has been computed as $C\left(\bar{y}(-1), \bar{y}^{\prime}(-1)\right)=(-1)^{B\left(\bar{y}, \bar{y}^{\prime}\right)}$. Thus, we find that $C\left(\bar{y}(-1), \bar{y}^{\prime}(-1)\right)=1$ for all $\bar{y}^{\prime} \in \overline{Y^{\Gamma}}$ if and only if $B\left(y, y^{\prime}\right) \in 2 \mathbb{Z}$ for all representatives $y$ of all $\bar{y}^{\prime} \in \overline{Y^{\Gamma}}$. This occurs if and only if $B\left(y, y^{\prime}\right) \in 2 \mathbb{Z}$ for all $y^{\prime} \in Y^{\Gamma}$, that is, $y \in Y^{\Gamma \#}$.

Thus, $\bar{y}(-1) \in Z^{\dagger}(T)$ for $\bar{y} \in \overline{Y^{\Gamma}}$ if and only if $y \in Y^{\Gamma \#} \cap Y^{\Gamma}=Y^{\Gamma \# \Gamma}$.

Corollary 7.4. Let $\boldsymbol{T}^{\Gamma \#}$ be the real torus with cocharacter group $Y^{\Gamma \#}$. Suppose $T^{\Gamma \#}=\boldsymbol{T}^{\Gamma \#}(\mathbb{R})$. Then the quotient $Z^{\dagger}(T) / T^{\circ}$ is isomorphic to $\operatorname{Im}\left(\pi_{0} T^{\Gamma \#} \rightarrow \pi_{0} T\right)$. 
Proof. The diagram

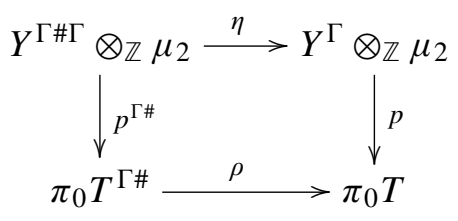

of finite abelian groups commutes. The previous proposition demonstrates that $Z^{\dagger}(T) / T^{\circ}$ can be identified with the image of $p \circ \eta$. The commutativity of the above diagram, together with the surjectivity of $p^{\Gamma \#}$, implies that this image is the same as the image of $\rho$.

The image of an isogeny. The inclusion $Y^{\#} \hookrightarrow Y$ of $\mathbb{Z}[\Gamma]$-modules corresponds, as in the nonarchimedean case, to an isogeny $\boldsymbol{\iota}: \boldsymbol{T}^{\#} \rightarrow \boldsymbol{T}$ of tori over $\mathbb{R}$. We are interested in the resulting continuous homomorphism $\imath: T^{\#} \rightarrow T$ of real Lie groups. Since $\iota$ is an isogeny, we find that $\iota\left(T^{\#}\right) \supset T^{\circ}$. Thus, in order to fully describe $\imath\left(T^{\#}\right)$, it suffices to determine the $\bar{y} \in \overline{Y^{\Gamma}}$ for which $\bar{y}(-1) \in \imath\left(T^{\#}\right)$.

Proposition 7.5. Let $\bar{y} \in \overline{Y^{\Gamma}}$. Then $\bar{y}(-1) \in \imath\left(T^{\#}\right)$ if and only if $\bar{y} \in \operatorname{Im}\left(\overline{Y^{\# \Gamma}} \rightarrow \overline{Y^{\Gamma}}\right)$. Proof. We find that $\bar{y}(-1) \in \imath\left(T^{\#}\right)$ if and only if there exists a $y \in Y^{\# \Gamma}$ that represents $\bar{y}$. The proposition follows.

Corollary 7.6. We can identify the quotient $\imath\left(T^{\#}\right) / T^{\circ}$ with $\operatorname{Im}\left(\pi_{0} T^{\#} \rightarrow \pi_{0} T\right)$.

Comparing the image of the isogeny $\imath$ to the group $Z^{\dagger}(T)$ yields a short exact sequence $1 \rightarrow \imath\left(T^{\#}\right) \rightarrow Z^{\dagger}(T) \rightarrow P^{\dagger} \rightarrow 1$, where we may identify the finite group

$$
P^{\dagger} \equiv \operatorname{Im}\left(\pi_{0} T^{\Gamma \#} \rightarrow \pi_{0} T\right) / \operatorname{Im}\left(\pi_{0} T^{\#} \rightarrow \pi_{0} T\right) .
$$

Parameterization. As for the case of nonarchimedean fields, we choose to parameterize the genuine irreducible representations of $\tilde{T}$ through a finite-to-one map and a description of the fibres. Over $\mathbb{R}$, the previous two sections imply that the space $\Phi_{\epsilon}(\tilde{T})$ can be identified (via Theorem 3.1 ) with the complex variety of genuine characters $\mathscr{L}_{\epsilon}(Z(\tilde{T}))$. This is a torsor for the complex algebraic group of characters $\mathscr{X}\left(Z^{\dagger}(T)\right)$. There is a short exact sequence

$$
1 \rightarrow \mathscr{X}\left(P^{\dagger}\right) \rightarrow \mathscr{X}\left(Z^{\dagger}(T)\right) \rightarrow H_{c}^{1}\left(\mathcal{W}_{\mathbb{R}}, \hat{\mathscr{T}}\right) / H_{c}^{1}\left(\mathcal{W}_{\mathbb{R}}, \hat{\mathscr{T}} \rightarrow \hat{\mathscr{T}}^{\#}\right) \rightarrow 1 .
$$

Hence:

Theorem 7.7. Suppose that we are given a metaplectic cover

$$
1 \rightarrow \mu_{n} \rightarrow \tilde{T} \rightarrow T \rightarrow 1
$$

of a real torus. Then, with the sublattices $Y^{\#} \subset Y^{\Gamma \#} \subset Y$ defined as before and the resulting isogenies $\boldsymbol{T}^{\#} \rightarrow \boldsymbol{T}^{\Gamma \#} \rightarrow \boldsymbol{T}$, we find that 
- there is a finite-to-one surjection

$$
\Phi: \Phi_{\epsilon}(\tilde{T}) \rightarrow H_{c}^{1}\left(\mathscr{W}_{\mathbb{R}}, \hat{\mathscr{T}}\right) / H_{c}^{1}\left(\mathscr{W}_{\mathbb{R}}, \hat{\mathscr{T}} \rightarrow \hat{\mathscr{T}}^{\#}\right)
$$

that intertwines the natural action of $H^{1}\left(\mathcal{W}_{\mathbb{R}}, \hat{\mathscr{T}}\right)$, and

- the fibres of this map are torsors for the finite group $\mathscr{X}\left(P^{\dagger}\right)$, where

$$
P^{\dagger}=\operatorname{Im}\left(\pi_{0} T^{\Gamma \#} \rightarrow \pi_{0} T\right) / \operatorname{Im}\left(\pi_{0} T^{\#} \rightarrow \pi_{0} T\right) .
$$

Note that this theorem is quite similar to the parameterization of $\Phi_{\epsilon}(\tilde{T})$ for tame covers of unramified tori over nonarchimedean local fields. The only difference is that points of residual tori are replaced by component groups.

\section{Acknowledgments}

I would like to thank Jeffrey Adams, Gordan Savin, and the referee for providing some advice and insight related to this paper. In addition, I am thankful for the hospitality and excellent working environment provided by the Hausdorff Institute for Mathematics in Bonn during the preparation of this paper. I thank the University of Michigan for its hospitality while this paper was being finished, during which Brian Conrad and Stephen DeBacker provided helpful conversations.

I am heavily indebted to Pierre Deligne, who read an early draft of this paper and gave extensive helpful comments. His generosity has led to significant improvements in presentation and content.

\section{References}

[Adams et al. 2007] J. Adams, D. Barbasch, A. Paul, P. Trapa, and D. A. Vogan, Jr., "Unitary Shimura correspondences for split real groups", J. Amer. Math. Soc. 20:3 (2007), 701-751. MR 2008i:22008 Zbl 1114.22009

[Borovoi 1998] M. Borovoi, Abelian Galois cohomology of reductive groups, Mem. Amer. Math. Soc. 132:626, Amer. Math. Soc., Providence, R.I., 1998. MR 98j:20061 Zbl 0918.20037

[Brylinski and Deligne 2001] J.-L. Brylinski and P. Deligne, "Central extensions of reductive groups by $\mathbf{K}_{2}$ ”, Publ. Math. Inst. Hautes Études Sci. 94 (2001), 5-85. MR 2004a:20049 Zbl 1093.20027

[Esnault et al. 1998] H. Esnault, B. Kahn, M. Levine, and E. Viehweg, "The Arason invariant and mod 2 algebraic cycles”, J. Amer. Math. Soc. 11:1 (1998), 73-118. MR 98d:14010 Zbl 1025.11009

[Hoffmann and Spitzweck 2007] N. Hoffmann and M. Spitzweck, "Homological algebra with locally compact abelian groups", Adv. Math. 212:2 (2007), 504-524. MR 2329311 Zbl 1123.22002

[Kazhdan and Patterson 1984] D. A. Kazhdan and S. J. Patterson, "Metaplectic forms", Inst. Hautes Études Sci. Publ. Math. 59 (1984), 35-142. MR 85g:22033 Zbl 0559.10026

[Kottwitz and Shelstad 1999] R. E. Kottwitz and D. Shelstad, Foundations of twisted endoscopy, Astérisque 255, Société Mathématique de France, Paris, 1999. MR 2000k:22024 Zbl 0958.22013

[Labesse 1985] J.-P. Labesse, "Cohomologie, L-groupes et fonctorialité”, Compositio Math. 55:2 (1985), 163-184. MR 86j:11117 Zbl 0595.12004 
[Lang 1956] S. Lang, “Algebraic groups over finite fields", Amer. J. Math. 78 (1956), 555-563. MR 19,174a Zbl 0073.37901

[Langlands 1997] R. P. Langlands, "Representations of abelian algebraic groups", Pacific J. Math. Special Issue (1997), 231-250. MR 99b:11125

[Milnor 1971] J. Milnor, Introduction to algebraic K-theory, Annals of Mathematics Studies 72, Princeton University Press, 1971. MR 50 \#2304 Zbl 0237.18005

[Moore 1964] C. C. Moore, "Extensions and low dimensional cohomology theory of locally compact groups. I, II”, Trans. Amer. Math. Soc. 113 (1964), 40-63. MR 30 \#2106 Zbl 0131.26902

[Ono 1961] T. Ono, "Arithmetic of algebraic tori”, Ann. of Math. (2) 74 (1961), 101-139. MR 23 \#A1640 Zbl 0119.27801

[Quillen 1973] D. Quillen, "Higher algebraic K-theory, I", pp. 85-147 in Algebraic K-theory, I: Higher $K$-theories (Seattle, WA, 1972), edited by H. Bass, Lecture Notes in Math. 341, Springer, Berlin, 1973. MR 49 \#2895 Zbl 0292.18004

[Savin 2004] G. Savin, “On unramified representations of covering groups”, J. Reine Angew. Math. 566 (2004), 111-134. MR 2005a:22014 Zbl 1032.22006

[Tate 1979] J. Tate, "Number theoretic background", pp. 3-26 in Automorphic forms, representations and L-functions, II (Corvallis, OR, 1977), edited by A. Borel and W. Casselman, Proc. Sympos. Pure Math. 33, Amer. Math. Soc., Providence, R.I., 1979. MR 80m:12009 Zbl 0422.12007

Received November 7, 2008.

\author{
Martin H. Weissman \\ DePaRTMENT OF MATHEMATICS \\ UNIVERSITY OF CALIFORNIA \\ SANTA CRUZ, CA 95064 \\ UNITED STATES \\ weissman@ucsc.edu \\ http://people.ucsc.edu/ weissman/
}

